\title{
Redheffer Representations and Relaxed Commutant Lifting
}

\author{
S. ter Horst
}

Received: 11 October 2009 / Accepted: 18 January 2010 / Published online: 9 February 2010

(C) The Author(s) 2010. This article is published with open access at Springerlink.com

\begin{abstract}
It is well known that the solutions of a (relaxed) commutant lifting problem can be described via a linear fractional representation of the Redheffer type. The coefficients of such Redheffer representations are analytic operator-valued functions defined on the unit disc $\mathbb{D}$ of the complex plane. In this paper we consider the converse question. Given a Redheffer representation, necessary and sufficient conditions on the coefficients are obtained guaranteeing the representation to appear in the description of the solutions to some relaxed commutant lifting problem. In addition, a result concerning a form of non-uniqueness appearing in the Redheffer representations under consideration and an harmonic maximal principle, generalizing a result of A. Biswas, are proved. The latter two results can be stated both on the relaxed commutant lifting as well as on the Redheffer representation level.
\end{abstract}

Keywords Relaxed commutant lifting - Linear fractional Redheffer representations · Operator-valued functions · Harmonic maximum principle

Mathematics Subject Classification (2000) $\quad$ Primary 47A56 - 47A20;

Secondary 47A57 $\cdot 47 \mathrm{~A} 48$

\section{Introduction}

Linear fractional representations have been used for describing solutions to metric constrained interpolation problems since the work of Adamyan-Arov-Kreĭn $[3,4]$,

Communicated by Daniel Alpay.

S. ter Horst $(\bowtie)$

Department of Mathematics, Utrecht University, Budapestlaan 6,

3584 CD Utrecht, The Netherlands

e-mail: s.terhorst@uu.nl 
and later appeared in the encompassing commutant lifting theory of Sz.-Nagy-Foiaş [26] and D. Sarason [30]; cf., [12,13]. They also play an important role in linear system theory [33]. The topic of the present paper is the class of linear fractional representation that appears in the context of the description of the solutions to the relaxed commutant lifting problem obtained in [20]. Such representations are called Redheffer representations, after the work of R. Redheffer [28,29].

Before we can introduce this class of representations, we require some definitions and notations. Let $\mathcal{U}$ and $\mathcal{Y}$ be Hilbert spaces. With the symbol $\mathbf{H}^{\infty}(\mathcal{U}, \mathcal{Y})$ we denote the set of uniformly bounded analytic functions on the open unit disc $\mathbb{D}$ with values in $\mathscr{L}(\mathcal{U}, \mathcal{Y})$. Here, as usual, we write $\mathscr{L}(\mathcal{U}, \mathcal{Y})$ for the space of operators from $\mathcal{U}$ into $\mathcal{Y}$. By definition, an operator is assumed to be linear and bounded. The $\operatorname{set} \mathbf{H}^{\infty}(\mathcal{U}, \mathcal{Y})$ is a Banach space with respect to the supremum norm \|\|$_{\infty}$; its closed unit ball is denoted by $\mathbf{S}(\mathcal{U}, \mathcal{Y})$ and called the Schur class associated with $\mathcal{U}$ and $\mathcal{Y}$. Functions in $\mathbf{S}(\mathcal{U}, \mathcal{Y})$ are called Schur class functions. It is well known that a function $F$ in $\mathbf{H}^{\infty}(\mathcal{U}, \mathcal{Y})$ defines a multiplication operator $M_{F}$ between the Hardy spaces $H^{2}(\mathcal{U})$ and $H^{2}(\mathcal{Y})$, and that $\|F\|_{\infty}$ is equal to the operator norm of $M_{F}$ in $\mathscr{L}\left(H^{2}(\mathcal{U}), H^{2}(\mathcal{Y})\right)$. We further define $\mathbf{H}^{2}(\mathcal{U}, \mathcal{Y})$ to be the set of analytic functions $H$ on $\mathbb{D}$ whose values are in $\mathscr{L}(\mathcal{U}, \mathcal{Y})$ with the property that the formula

$$
\left(\Gamma_{H} u\right)(\lambda)=H(\lambda) u \quad(u \in \mathcal{U}, \lambda \in \mathbb{D})
$$

defines an operator from $\mathcal{U}$ into the Hardy space $H^{2}(\mathcal{Y})$. The $\operatorname{set} \mathbf{H}^{2}(\mathcal{U}, \mathcal{Y})$ is a Banach space with norm $\|H\|=\left\|\Gamma_{H}\right\|$, i.e., the norm of $H \in \mathbf{H}^{2}(\mathcal{U}, \mathcal{Y})$ is equal to the operator norm of the associated operator $\Gamma_{H}$. We write $\mathbf{H}_{\text {ball }}^{2}(\mathcal{U}, \mathcal{Y})$ for the closed unit ball of $\mathbf{H}^{2}(\mathcal{U}, \mathcal{Y})$. Note that, conversely, any operator $\Gamma \in \mathscr{L}\left(\mathcal{U}, H^{2}(\mathcal{Y})\right)$ defines a function $H \in \mathbf{H}^{2}(\mathcal{U}, \mathcal{Y})$ via the same identity $H(\lambda) u=(\Gamma u)(\lambda)$.

Now let $\mathcal{U}, \mathcal{Y}, \mathcal{E}$ and $\mathcal{E}^{\prime}$ be Hilbert spaces and $\Psi$ an operator-valued function on $\mathbb{D}$ that decomposes as $\Psi=\left[\begin{array}{ll}\Psi_{1,1} & \Psi_{1,2} \\ \Psi_{2,1} & \Psi_{2,2}\end{array}\right]$ so that $\Psi_{1,1}(0)=0$ and

$$
\Psi_{1,1} \in \mathbf{S}\left(\mathcal{E}^{\prime}, \mathcal{E}\right), \quad \Psi_{1,2} \in \mathbf{H}_{\text {ball }}^{2}(\mathcal{U}, \mathcal{E}), \quad \Psi_{2,1} \in \mathbf{S}\left(\mathcal{E}^{\prime}, \mathcal{Y}\right), \quad \Psi_{2,2} \in \mathbf{H}_{\text {ball }}^{2}(\mathcal{U}, \mathcal{Y})
$$

The Redheffer representation associated with $\Psi$ is the map $V \mapsto \mathscr{R}_{\Psi}[V]$ given by

$$
\mathscr{R}_{\Psi}[V](\lambda)=\Psi_{2,2}(\lambda)+\Psi_{2,1}(\lambda) V(\lambda)\left(I-\Psi_{1,1}(\lambda) V(\lambda)\right)^{-1} \Psi_{1,2}(\lambda)
$$

and defined for $V \in \mathbf{S}\left(\mathcal{E}, \mathcal{E}^{\prime}\right)$. The functions $\Psi_{1,1}, \Psi_{1,2}, \Psi_{2,1}$ and $\Psi_{2,2}$ are referred to as the associated Redheffer coefficients. In addition, we shall assume the coefficient matrix

$$
\left[\begin{array}{ll}
M_{\Psi_{1,1}} & \Gamma_{\Psi_{1,2}} \\
M_{\Psi_{2,1}} & \Gamma_{\Psi_{2,2}}
\end{array}\right]\left[\begin{array}{l}
H^{2}\left(\mathcal{E}^{\prime}\right) \\
\mathcal{U}
\end{array}\right] \rightarrow\left[\begin{array}{l}
H^{2}(\mathcal{E}) \\
H^{2}(\mathcal{Y})
\end{array}\right]
$$

to be a contraction, or, more often a co-isometry. It is then the case that the Redheffer representation $\mathscr{R}_{\Psi}$ maps $\mathbf{S}\left(\mathcal{E}, \mathcal{E}^{\prime}\right)$ into $\mathbf{H}_{\text {ball }}^{2}(\mathcal{U}, \mathcal{Y})$ (see Proposition1 4.1). Note 
that this includes the more conventional class of Redheffer representations where the function $\Psi$ is a Schur class function itself, in which case $\mathcal{R}_{\Psi}$ maps $\mathbf{S}\left(\mathcal{E}, \mathcal{E}^{\prime}\right)$ into $\mathbf{S}(\mathcal{U}, \mathcal{Y})$.

The relaxed commutant lifting problem was introduced in [14]; the developed theory extends the classical commutant lifting theory [26], as well as the Treil-Volberg commutant lifting setting [31] and the weighted version of [9]. See [11] for an application in filterbank design. In [14] a particular (central) solution is given; while descriptions of all solutions are obtained in $[16,17,20,22,25]$. The starting point for the relaxed commutant lifting problem is a lifting data set $\left\{A, T^{\prime}, U^{\prime}, R, Q\right\}$ consisting of five Hilbert space operators: the operator $A$ is a contraction mapping $\mathcal{H}$ into $\mathcal{H}^{\prime}$, the operator $U^{\prime}$ on $\mathcal{K}^{\prime}$ is a minimal isometric lifting of the contraction $T^{\prime}$ on $\mathcal{H}^{\prime}$, i.e., $U^{\prime}$ is an isometry on $\mathcal{K}^{\prime}$ with $\mathcal{H}^{\prime} \subset \mathcal{K}^{\prime}$ being cyclic for $U^{\prime}$ and $\Pi_{\mathcal{H}^{\prime}} U^{\prime}=T^{\prime} \Pi_{\mathcal{H}^{\prime}}$, and $R$ and $Q$ are operators from $\mathcal{H}_{0}$ to $\mathcal{H}$, satisfying

$$
T^{\prime} A R=A Q \text { and } R^{*} R \leq Q^{*} Q
$$

Given this data set the relaxed commutant lifting problem is to describe a (all) contraction(s) $B$ from $\mathcal{H}$ to $\mathcal{K}^{\prime}$ such that

$$
\Pi_{\mathcal{H}^{\prime}} B=A \text { and } \quad U^{\prime} B R=B Q
$$

Here we follow the convention that for a subspace $\mathcal{V}$ of a Hilbert space $\mathcal{W}$ the symbol $\Pi_{\mathcal{V}}$ stands for the orthogonal projection from $\mathcal{W}$ onto $\mathcal{V}$ viewed as an operator from $\mathcal{W}$ onto $\mathcal{V}$, whereas $P_{\mathcal{V}}$ is used for the orthogonal projection from $\mathcal{W}$ onto $\mathcal{V}$ as an operator from $\mathcal{W}$ into $\mathcal{W}$. A contraction $B$ from $\mathcal{H}$ into $\mathcal{K}^{\prime}$ that satisfies (0.6) is called a contractive interpolant for $\left\{A, T^{\prime}, U^{\prime}, R, Q\right\}$. The Treil-Volberg version appears when $R$ is the identity operator on $\mathcal{H}$, and thus $\mathcal{H}_{0}=\mathcal{H}$; for classical commutant lifting it is assumed in addition that $Q$ is an isometry.

Without loss of generality we may, and will, assume that the isometric lifting $U^{\prime}$ in $\left\{A, T^{\prime}, U^{\prime}, R, Q\right\}$ is the Sz.-Nagy Schäffer isometric lifting of $T^{\prime}$, i.e.,

$$
U^{\prime}=\left[\begin{array}{ll}
T^{\prime} & 0 \\
E_{\mathcal{D}_{T^{\prime}}} D_{T^{\prime}} & S_{\mathcal{D}_{T^{\prime}}}
\end{array}\right] \text { on }\left[\begin{array}{l}
\mathcal{H}^{\prime} \\
H^{2}\left(\mathcal{D}_{T^{\prime}}\right)
\end{array}\right]
$$

where we use the general notation $S_{\mathcal{U}}$ for the forward shift on the Hardy space $H^{2}(\mathcal{U})$, and $E_{\mathcal{U}}$ for the canonical embedding of $\mathcal{U}$ into the subspace of constant functions in $H^{2}(\mathcal{U})$, that is, $\left(E_{\mathcal{U}} u\right)(\lambda)=u$ for all $\lambda \in \mathbb{D}=\{\lambda \in \mathbb{C}:|\lambda|<1\}$ and $u \in \mathcal{U}$. Furthermore, as usual, given a contraction $N$, we write $D_{N}$ for the defect operator and $\mathcal{D}_{N}$ for the defect space of $N$, that is, $D_{N}$ is the positive square root of $I-N^{*} N$ and $\mathcal{D}_{N}$ is the closure of the range of $D_{N}$.

An initial step in the process of describing the solutions to a commutant lifting problem is the extraction of an operator from the data. In the case of the relaxed commutant lifting problem we obtain a contraction $\omega$ defined by

$$
\omega: \mathcal{F}=\overline{D_{A} Q \mathcal{H}_{0}} \rightarrow\left[\begin{array}{l}
\mathcal{D}_{T^{\prime}} \\
\mathcal{D}_{A}
\end{array}\right], \quad \omega D_{A} Q=\left[\begin{array}{l}
D_{T^{\prime}} A R \\
D_{A} R
\end{array}\right],
$$


which we refer to as the underlying contraction of $\left\{A, T^{\prime}, U^{\prime}, R, Q\right\}$ (see [14]). We write $\omega_{1}$ for the component of $\omega$ that maps $\mathcal{F}$ into $\mathcal{D}_{T^{\prime}}$ and $\omega_{2}$ for the component of $\omega$ that maps $\mathcal{F}$ into $\mathcal{D}_{A}$. In the classical commutant lifting setting, as well as in many of the examples considered in [14], we have $R^{*} R=Q^{*} Q$, which is equivalent to $\omega$ being an isometry.

Once the underlying contraction $\omega$ is obtained from the data, we set $\mathcal{G}=\mathcal{D}_{A} \ominus \mathcal{F}$ and write $\Pi_{\mathcal{F}}$ and $\Pi_{\mathcal{G}}$ for the orthogonal projections in $\mathscr{L}\left(\mathcal{D}_{A}, \mathcal{F}\right)$ and $\mathscr{L}\left(\mathcal{D}_{A}, \mathcal{G}\right)$, respectively. The symbols $\Pi_{\mathcal{D}_{A}}$ and $\Pi_{\mathcal{D}_{T^{\prime}}}$ will be used for the orthogonal projections from $\mathcal{D}_{A} \oplus \mathcal{D}_{T^{\prime}}$ onto $\mathcal{D}_{A}$, respectively $\mathcal{D}_{T^{\prime}}$. Next we define operator-valued functions $\Phi_{1,1}, \Phi_{1,2}, \Phi_{2,1}$ and $\Phi_{2,2}$ on $\mathbb{D}$ by

$$
\begin{aligned}
& \Phi_{1,1}(\lambda)=\lambda \Pi_{\mathcal{G}}\left(I_{\mathcal{D}_{A}}-\lambda \omega_{2} \Pi_{\mathcal{F}}\right)^{-1} \Pi_{\mathcal{D}_{A}} D_{\omega^{*}}, \\
& \Phi_{1,2}(\lambda)=\Pi_{\mathcal{G}}\left(I_{\mathcal{D}_{A}}-\lambda \omega_{2} \Pi_{\mathcal{F}}\right)^{-1}, \\
& \Phi_{2,1}(\lambda)=\Pi_{\mathcal{D}_{T^{\prime}}} D_{\omega^{*}}+\lambda \omega_{1} \Pi_{\mathcal{F}}\left(I_{\mathcal{D}_{A}}-\lambda \omega_{2} \Pi_{\mathcal{F}}\right)^{-1} \Pi_{\mathcal{D}_{A}} D_{\omega^{*}}, \quad(\lambda \in \mathbb{D}) \\
& \Phi_{2,2}(\lambda)=\omega_{1} \Pi_{\mathcal{F}}\left(I_{\mathcal{D}_{A}}-\lambda \omega_{2} \Pi_{\mathcal{F}}\right)^{-1} .
\end{aligned}
$$

and put

$$
\Phi=\left[\begin{array}{ll}
\Phi_{1,1} & \Phi_{1,2} \\
\Phi_{2,1} & \Phi_{2,2}
\end{array}\right]
$$

The set of all contractive interpolants is then described by the following theorem; see Theorem 5.1.1 in [20].

Theorem 0.1 Let $\left\{A, T^{\prime}, U^{\prime}, R, Q\right\}$ be a lifting data set with underlying contraction $\omega$. Set $\mathcal{G}=\mathcal{D}_{A} \ominus D_{A} Q \mathcal{H}_{0}$ and define $\Phi_{1,1}, \Phi_{1,2}, \Phi_{2,1}$ and $\Phi_{2,2}$ by (0.8). Then

$$
\begin{aligned}
& \Phi_{1,1} \in \mathbf{S}\left(\mathcal{D}_{\omega^{*}}, \mathcal{G}\right), \quad \Phi_{1,2} \in \mathbf{H}_{\text {ball }}^{2}\left(\mathcal{D}_{A}, \mathcal{G}\right), \quad \Phi_{2,1} \in \mathbf{S}\left(\mathcal{D}_{\omega^{*}}, \mathcal{D}_{T^{\prime}}\right) \\
& \Phi_{2,2} \in \mathbf{H}_{\text {ball }}^{2}\left(\mathcal{D}_{A}, \mathcal{D}_{T^{\prime}}\right)
\end{aligned}
$$

$\Phi_{1,1}(0)=0$ and the coefficient matrix

$$
K_{0}=\left[\begin{array}{ll}
M_{\Phi_{1,1}} & \Gamma_{\Phi_{1,2}} \\
M_{\Phi_{2,1}} & \Gamma_{\Phi_{2,2}}
\end{array}\right]:\left[\begin{array}{l}
H^{2}\left(\mathcal{D}_{\omega^{*}}\right) \\
\mathcal{D}_{A}
\end{array}\right] \rightarrow\left[\begin{array}{l}
H^{2}(\mathcal{G}) \\
H^{2}\left(\mathcal{D}_{\omega^{*}}\right)
\end{array}\right]
$$

is a co-isometry. Moreover, set $H_{V}=\mathscr{R}_{\Phi}[V] \in \mathbf{H}_{\text {ball }}^{2}\left(\mathcal{D}_{A}, \mathcal{D}_{T^{\prime}}\right)$ for any $V$ in $\mathbf{S}\left(\mathcal{G}, \mathcal{D}_{\omega^{*}}\right)$ and define

$$
B_{V}=\left[\begin{array}{l}
A \\
\Gamma_{H_{V}} D_{A}
\end{array}\right]: \mathcal{H} \rightarrow\left[\begin{array}{l}
\mathcal{H}^{\prime} \\
H^{2}\left(\mathcal{D}_{T^{\prime}}\right)
\end{array}\right]
$$

Then $B_{V}$ is a contractive interpolant for $\left\{A, T^{\prime}, U^{\prime}, R, Q\right\}$, and all contractive interpolants are obtained in this way. Finally, $K_{0}$ is unitary if and only if $\omega$ is an isometry and $\omega_{2} \Pi_{\mathcal{F}}$ on $\mathcal{D}_{A}$ is strongly stable (i.e., $\lim _{n \rightarrow \infty}\left(\omega_{2} \Pi_{\mathcal{F}}\right)^{n} u=0$ for each $u \in \mathcal{D}_{A}$ ). 
When we take $V=\underline{0}$, the constant function whose value is the zero operator, we see that a particular contractive interpolant is obtained by taking $H_{V}=\Phi_{2,2}$ in (0.11); this contractive interpolant is called the central contractive interpolant and was already obtained in [14].

The subscript 0 in $K_{0}$ also refers to the zero function $V=\underline{0}$; the operator $K_{0}$ is a special case of the operator $K_{V}$ defined in (4.5).

One point where the Redheffer representation of Theorem 0.1 is distinctively different from the one in the classical commutant lifting setting is that the map $\mathscr{R}_{\Phi}$ is, in general, not one-to-one, and thus the same holds true for the map $V \mapsto B_{V}$. This property is inherited from the Schur representation of [17] (from which the Redheffer representation of Theorem 0.1 is deduced in [20]) where the same phenomenon occurs. We return to this issue later in the introduction.

Theorem 0.1 raises the question how the set of Redheffer representations $\mathscr{R}_{\Phi}$ with $\Phi$ obtained from a relaxed commutant lifting problem is situated in the set of all Redheffer representations $\mathscr{R}_{\Psi}$ with coefficients as in (0.2). We further restrict the set of Redheffer representations $\mathscr{R}_{\Psi}$ by demanding that $\Psi_{1,1}(0)=0$ and that the coefficient matrix (0.4) is a co-isometry, as this is a natural restriction based on the result of Theorem 0.1.

To be precise, we will consider the following question: Given a Redheffer representation $\mathcal{R}_{\Psi}$ with coefficients as a in (0.2), with $\Psi_{1,1}(0)=0$ and (0.4) a co-isometry, when does there exist a lifting data set $\left\{A, T^{\prime}, U^{\prime}, R, Q\right\}$ so that the set of all contractive interpolants for the associated relaxed commutant lifting problem is given by

$$
\left\{B_{V}=\left[\begin{array}{l}
A \\
\Gamma_{H_{V}} D_{A}
\end{array}\right] \mid H_{V}=\mathscr{R}_{\Psi}[V] \quad \text { for some } V \in \mathbf{S}\left(\mathcal{E}, \mathcal{E}^{\prime}\right)\right\} .
$$

If $\left\{A, T^{\prime}, U^{\prime}, R, Q\right\}$ has this property, we say it is a lifting data set associated with the Redheffer representation $\mathscr{R}_{\Psi}$. Note that the lifting data set $\left\{A, T^{\prime}, U^{\prime}, R, Q\right\}$ should satisfy $\mathcal{D}_{A}=\mathcal{U}$ and $\mathcal{D}_{T^{\prime}}=\mathcal{Y}$.

There is a different kind of inverse problem for interpolation and commutant lifting problems that goes back to the work of Adamjan-Arov-Krel̆n [3,4] on the Nehari problem, and has since been considered in various settings [5-7,23,24,32], see also [2]. In its original form, for the Nehari problem, this inverse problem takes the form of the question which $\gamma$-generating pairs are also Nehari pairs. An extension to the classical commutant lifting setting is posed and solved by J. A. Ball and A. Kheifets in [7]. For such problems the data of the considered problem is known, and a Redheffer representation is given for which it is also known that its range forms a subset of the set of all solutions for the problem defined by the given data. The inverse problem is then to determine if the solutions generated by the Redheffer transformation are in fact all solutions. In the present paper, the inverse problem is to construct a data set so that the given Redheffer representation provides all solutions. As a consequence, the solution criteria we find here looks quite different from the one obtained in [7].

Somewhat surprisingly, it turns out that for the problem considered here, it is always possible to find an associated lifting data set. 
Lifting data set
$\left\{A, T^{\prime}, U^{\prime}, R, Q\right\}$$\Longrightarrow \begin{gathered}\text { Underlying contraction } \\ \omega\end{gathered} \Longrightarrow \begin{aligned} & \text { Redheffer coefficients } \\ & \Phi_{1,1}, \Phi_{1,2}, \Phi_{2,1}, \Phi_{2,2}\end{aligned}$

Fig. 1 Lifting data set to Redheffer coefficients

Theorem 0.2 Let $\mathscr{R}_{\Psi}$ be a Redheffer representation with coefficients as a in (0.2) so that $\Psi_{1,1}(0)=0$ and the coefficient matrix (0.4) is a co-isometry. Then there exists a lifting data set $\left\{A, T^{\prime}, U^{\prime}, R, Q\right\}$ associated with $\mathscr{R}_{\Psi}$. More precisely, if $\Phi_{1,1}, \Phi_{1,2}$, $\Phi_{2,1}$ and $\Phi_{2,2}$ are the Redheffer coefficients associated with $\left\{A, T^{\prime}, U^{\prime}, R, Q\right\}$ via $(0.8)$, then there exist a unitary operator $\psi$ mapping $\mathcal{E}$ onto $\mathcal{G}$ and a co-isometry $\varphi$ from $\mathcal{E}^{\prime}$ onto $\mathcal{D}_{\omega^{*}}$ such that for each $\lambda \in \mathbb{D}$

$$
\left[\begin{array}{ll}
\psi & 0 \\
0 & I \mathcal{Y}
\end{array}\right]\left[\begin{array}{ll}
\Psi_{1,1}(\lambda) & \Psi_{1,2}(\lambda) \\
\Psi_{2,1}(\lambda) & \Psi_{2,2}(\lambda)
\end{array}\right]=\left[\begin{array}{ll}
\Phi_{1,1}(\lambda) & \Phi_{1,2}(\lambda) \\
\Phi_{2,1}(\lambda) & \Phi_{2,2}(\lambda)
\end{array}\right]\left[\begin{array}{ll}
\varphi & 0 \\
0 & I_{\mathcal{U}}
\end{array}\right]
$$

Moreover, if in addition the coefficient matrix (0.4) is unitary, then $\varphi$ is unitary.

Since the Redheffer coefficients ( 0.8$)$ are completely determined by the underlying contraction $\omega$, we see that the construction goes along the path shown in Fig. 1. It was already observed in [18] that any contraction $\omega$ of the form

$$
\omega=\left[\begin{array}{l}
\omega_{1} \\
\omega_{2}
\end{array}\right]: \mathcal{F} \rightarrow\left[\begin{array}{l}
\mathcal{Y} \\
\mathcal{U}
\end{array}\right], \quad \mathcal{F} \subset \mathcal{U}
$$

appears as the underlying contraction of some lifting data set. We prove Theorem 0.2 in Sect. 2 below by extracting a contraction $\omega$ of the form $(0.14)$ from the Redheffer coefficients.

Theorem 0.2 guarantees the existence of a lifting data set with the required properties. Moreover, as we will see in the course of the proof in Sect. 2 below, the lifting data set can in fact be constructed explicitly from the given coefficients. We already observed that for an associated lifting data set $\left\{A, T^{\prime}, U^{\prime}, R, Q\right\}$ we have $\mathcal{D}_{A}=\mathcal{U}$ and $\mathcal{D}_{T^{\prime}}=\mathcal{Y}$. In addition, the space $\mathcal{F}$ on which the underlying contraction $\omega$ associated with the lifting data set is defined and the unitary operator $\psi$ can also be extracted from the given coefficients. Indeed, from the definition of $\Phi_{1,2}$ in $(0.8)$ and the relation (0.13) it follows that $\mathcal{F}=\operatorname{Ker} \Psi_{1,2}(0)$, while $\psi^{*}=\Psi_{1,2}(0) \mid \mathcal{U} \ominus \mathcal{F}$. Hence the Hilbert spaces between which the contraction $\omega$ underlying $\left\{A, T^{\prime}, U^{\prime}, R, Q\right\}$ acts are determined by the Redheffer coefficients.

Note that, as one can see from (0.13), $\Psi_{1,1}, \Psi_{1,2}, \Psi_{2,1}$ and $\Psi_{2,2}$ are, in general, not the actual coefficients associated with the constructed data set, not even up to unitary transformations on the spaces $\mathcal{E}$ and $\mathcal{E}^{\prime}$, unless the operator $\varphi$ is unitary. If $\varphi$ is not unitary and $\mathcal{E}_{0}^{\prime}=\operatorname{Ker} \varphi$, then $\left.\Psi_{1,1}(\lambda)\right|_{\mathcal{E}_{0}^{\prime}}=0$ and $\left.\Psi_{2,1}(\lambda)\right|_{\mathcal{E}_{0}^{\prime}}=0$ for each $\lambda \in \mathbb{D}$, which only adds to the non-uniqueness in the Redheffer representation: For $V, V^{\prime} \in \mathbf{S}\left(\mathcal{E}, \mathcal{E}^{\prime}\right)$ with $P_{\mathcal{E}^{\prime} \ominus \mathcal{E}_{0}^{\prime}} V(\lambda)=P_{\mathcal{E}^{\prime} \ominus \mathcal{E}_{0}^{\prime}} V^{\prime}(\lambda)$ for all $\lambda \in \mathbb{D}$ we have $H_{V}=H_{V^{\prime}}$.

The two steps in the construction, lifting data set to underlying contraction and underlying contraction to Redheffer coefficients, are in general not one-to-one. Many lifting data sets can have the same underlying contraction and for each admissible set 
of Redheffer coefficients there can be more than one underlying contraction. However, it turns out that under some additional assumptions all contractions $\omega$ that define a fixed set of Redheffer coefficients via (0.8) are unique up to a unitary transformation; see Proposition 0.3 below.

Let $\omega^{\prime}$ be the underlying contraction of another lifting data set associated with the Redheffer coefficients (0.2), and assume that the conditions of Theorem 0.2 are met. Then $\omega^{\prime}$ also maps $\mathcal{F}=\operatorname{Ker} \Psi_{1,2}(0)$ into $\mathcal{Y} \oplus \mathcal{U}$. We say that $\omega$ and $\omega^{\prime}$ are unitarily equivalent if there exists a unitary operator $\Theta$ on $\mathcal{U}$ for which $\mathcal{F}$ is a reducing subspace $\left(\Theta \mathcal{F} \subset \mathcal{F}\right.$ and $\left.\Theta^{*} \mathcal{F} \subset \mathcal{F}\right)$ such that

$$
\omega_{1} \Pi_{\mathcal{F}} \Theta=\omega_{1}^{\prime} \Pi_{\mathcal{F}} \quad \text { and } \quad \omega_{2} \Pi_{\mathcal{F}} \Lambda=\Lambda \omega_{2}^{\prime} \Pi_{\mathcal{F}}
$$

It is not difficult to see that unitarily equivalent underlying contractions define the same Redheffer coefficients via (0.8). If, in addition, the coefficient matrix (0.4) is unitary, then the converse is also true.

Proposition 0.3 Let $\left\{A, T^{\prime}, U^{\prime}, R, Q\right\}$ and $\left\{\widetilde{A}, \widetilde{T}^{\prime}, \widetilde{U^{\prime}}, \widetilde{R}, \widetilde{Q}\right\}$ be lifting data sets associated with Redheffer coefficients (0.2) that satisfy the conditions of Theorem 0.2. Assume that the coefficient matrix (0.4) is unitary. Then the underlying contractions of $\left\{A, T^{\prime}, U^{\prime}, R, Q\right\}$ and $\left\{\widetilde{A}, \widetilde{T}^{\prime}, \widetilde{U}^{\prime}, \widetilde{R}, \widetilde{Q}\right\}$ are unitarily equivalent.

Since the steps in the construction are in general not one-to-one, it is of interest which properties are invariant under these steps. Two invariants are known:

(i) $R^{*} R=Q^{*} Q \Longleftrightarrow \omega$ is an isometry;

$$
\begin{array}{ll}
\omega \text { is an isometry and } & \Longleftrightarrow \begin{array}{l}
\text { the coefficient matrix } \\
\omega_{2} \Pi_{\mathcal{F}} \text { is strongly stable }
\end{array} \\
\text { is unitary. }
\end{array}
$$

If the operators in the lifting data set satisfy: $R^{*} R=Q^{*} Q,\|A\|<1$ and $R$ is left invertible (or equivalently $R^{*} R$ is invertible), then we are in case (ii), but these conditions on the lifting data set are only sufficient; in fact, any $\omega$ of the form (0.14) is the underlying contraction of a lifting data set with $A$ a co-isometry (see the proof of Theorem 0.2 below).

We now return to the topic of the non-uniqueness in the Redheffer representation of Theorem 0.1 . Let $B$ be a contractive interpolant for the lifting data set $\left\{A, T^{\prime}, U^{\prime}, R, Q\right\}$, and define

$$
\mathscr{V}_{B}=\left\{V \in \mathbf{S}\left(\mathcal{G}, \mathcal{D}_{\omega^{*}}\right) \mid B=B_{V}\right\}
$$

In [17] necessary and sufficient conditions were obtained that guarantee that the set $\mathscr{V}_{B}$ consists of one element only. To state the criteria some additional notation is required. Assume that $B$ is given in the form $(0.11)$, i.e., we have a contraction $\Gamma \in \mathscr{L}\left(\mathcal{D}_{A}, H^{2}\left(\mathcal{D}_{T^{\prime}}\right)\right)$ such that

$$
B=\left[\begin{array}{l}
A \\
\Gamma D_{A}
\end{array}\right] .
$$


As in [17] we define a contraction $\omega_{B}$ by

$$
\omega_{B}: \mathcal{F}_{B}=\overline{D_{\Gamma} \mathcal{F}} \rightarrow \mathcal{D}_{\Gamma}, \quad \omega_{B} D_{\Gamma} \mid \mathcal{F}=D_{\Gamma} \omega_{2}
$$

It turns out that $\omega_{B}$ is an isometry if and only if $\omega$ is an isometry, i.e., if and only if $R^{*} R=Q^{*} Q$. Hence the statement " $\omega_{B}$ is an isometry" holds true, or not, independent of the contractive interpolant $B$ in question. Set $\mathcal{G}_{B}=\mathcal{D}_{\Gamma} \ominus \mathcal{F}_{B}$. Using this contraction $\omega_{B}$ a one-to-one map from the Schur class $\mathbf{S}\left(\mathcal{G}_{B}, \mathcal{D}_{\omega_{B}^{*}}\right)$ onto the set $\mathscr{V}_{B}$ can be constructed; see Theorem 1.2 and the subsequent paragraph in [17]. Since $\mathcal{G}_{B}=\{0\}$ if and only if $\mathcal{F}_{B}=\mathcal{D}_{\Gamma_{H}}$ and $\mathcal{D}_{\omega_{B}^{*}}=\{0\}$ if and only if $\omega_{B}$ is a co-isometry, we have the following criterion for the set $\mathscr{V}_{B}$ to be a singleton.

Proposition 0.4 Let $B$ be a contractive interpolant for $\left\{A, T^{\prime}, U^{\prime}, R, Q\right\}$. Let $\omega_{B}$ be the contraction from $\mathcal{F}_{H}$ into $\mathcal{D}_{\Gamma_{H}}$ defined by (0.16). Then the set $\mathscr{V}_{B}$ is a singleton if and only if $\mathcal{F}_{B}=\mathcal{D}_{\Gamma_{H}}$ or $\omega_{B}$ is a co-isometry.

Proposition 0.4 gives necessary and sufficient conditions under which the set $\mathscr{V}_{B}$ consists of just one element for each contractive interpolant $B$ individually. It is not clear how to derive necessary and sufficient conditions for the map $V \mapsto B_{V}$ to be oneto-one from this result; sufficient conditions obtained in the literature are: $\mathcal{F}=\mathcal{D}_{A}$, $\omega$ is a co-isometry or $\omega$ is an isometry and $\overline{\omega_{2} \mathcal{F}}=\mathcal{D}_{A}$; see Proposition 4.2.8 in [20] and the proof of Theorem 1.3 in [17]. However, the fact that the contraction $\omega_{B}$ is an isometry either for all contractive interpolants $B$ or for none gives hope that a global result may be obtainable.

The next theorem presents a global result when we restrict to the set of contractive interpolants $B_{V}$ where $V$ is from the open ball $\mathbf{S}_{0}\left(\mathcal{G}, \mathcal{D}_{\omega^{*}}\right)$ of $\mathbf{H}^{\infty}\left(\mathcal{G}, \mathcal{D}_{\omega^{*}}\right)$, that is, from the set

$$
\mathbf{S}_{0}\left(\mathcal{G}, \mathcal{D}_{\omega^{*}}\right):=\left\{V \in \mathbf{S}\left(\mathcal{G}, \mathcal{D}_{\omega^{*}}\right):\|V\|_{\infty}<1\right\}
$$

and under the additional assumptions the underlying contraction $\omega$ is an isometry and $\omega_{2} \Pi_{\mathcal{F}}$ is strongly stable. The latter happens in many of the examples considered in $[13,14]$ if the operator $A$ is a strict contraction.

Theorem 0.5 Let $\left\{A, T^{\prime}, U^{\prime}, R, Q\right\}$ be a lifting data set such that the underlying contraction $\omega$ is an isometry and $\omega_{2} \Pi_{\mathcal{F}}$ on $\mathcal{D}_{A}$ is strongly stable. Then for each $V \in \mathbf{S}_{0}\left(\mathcal{G}, \mathcal{D}_{\omega^{*}}\right)$ we have $\mathscr{V}_{B_{V}}=\{V\}$ if and only if

$$
\Gamma_{\Phi_{1,2}} \mathcal{D}_{A}=\left\{\Pi_{\mathcal{G}}\left(I-\lambda \omega_{2} \Pi_{\mathcal{F}}\right) u \mid u \in \mathcal{D}_{A}\right\}
$$

is dense in $H^{2}(\mathcal{G})$. In particular, if $\mathscr{V}_{B_{V}}=\{V\}$ for some $V \in \mathbf{S}_{0}\left(\mathcal{G}, \mathcal{D}_{\omega^{*}}\right)$, then $\mathscr{V}_{B_{V}}=\{V\}$ for all $V \in \mathbf{S}_{0}\left(\mathcal{G}, \mathcal{D}_{\omega^{*}}\right)$.

Given a lifting data set $\left\{A, T^{\prime}, U^{\prime}, R, Q\right\}$ that satisfies the requirement of Theorem 0.5 , it follows that the Redheffer representation of Theorem 0.1 cannot be oneto-one in case $\mathcal{D}_{A}$ is finite dimensional, unless $\mathcal{G}=\{0\}$. This occurs, for instance, in the examples of the relaxations of classical interpolation problems introduced in [14], 
where the common ingredient is that $R$ and $Q$ are operators from $\mathcal{V}^{n-1}$ into $\mathcal{V}^{n}$ of the form

$$
R=\left[\begin{array}{l}
I_{\mathcal{V}^{n-1}} \\
0
\end{array}\right] \text { and } Q=\left[\begin{array}{l}
0 \\
I_{\mathcal{V}^{n-1}}
\end{array}\right]
$$

assuming that $\operatorname{dim} \mathcal{V}<\infty$ and $\|A\|<1$.

Another example of a global result for the set of contractive interpolants $B_{V}$ associated with $\mathbf{S}_{0}\left(\mathcal{G}, \mathcal{D}_{\omega^{*}}\right)$ is the harmonic maximum principle for the classical commutant lifting problem due to A. Biswas [8]. As a result of our investigations of Redheffer representations in Sect. 4 below we obtain the following extension of Theorem 3.1 from [8] to the relaxed commutant lifting setting.

Theorem 0.6 Given a lifting data set $\left\{A, T^{\prime}, U^{\prime}, R, Q\right\}$ we have:

(1) If there exists a $\widetilde{V} \in \mathbf{S}_{0}\left(\mathcal{G}, \mathcal{D}_{\omega^{*}}\right)$ so that $\left\|B_{\widetilde{V}}\right\|<1$, then $\left\|B_{V}\right\|<1$ for all $V \in \mathbf{S}_{0}\left(\mathcal{G}, \mathcal{D}_{\omega^{*}}\right)$.

(2) If there exists a $\widetilde{V} \in \mathbf{S}_{0}\left(\mathcal{G}, \mathcal{D}_{\omega^{*}}\right)$ so that $\left\|B_{\widetilde{V}}\right\|=1$, then $\left\|B_{V}\right\|=1$ for all $V \in \mathbf{S}_{0}\left(\mathcal{G}, \mathcal{D}_{\omega^{*}}\right)$.

(3) Moreover, for $V \in \mathbf{S}_{0}\left(\mathcal{G}, \mathcal{D}_{\omega^{*}}\right)$ and $\widetilde{V} \in \mathbf{S}\left(\mathcal{G}, \mathcal{D}_{\omega^{*}}\right)$ we have $\operatorname{Ker} D_{B_{V}} \subset$ $\operatorname{Ker} D_{B_{\widetilde{V}}}$. In particular, $\operatorname{Ker} D_{B_{V}}=\operatorname{Ker} D_{B_{\tilde{V}}}$ in case $\widetilde{V}, V \in \mathbf{S}_{0}\left(\mathcal{G}, \mathcal{D}_{\omega^{*}}\right)$.

When restricting to the classical commutant lifting setting, Part 2 coincides with Theorem 3.1 from [8]. Part 1 is just the contrapositive of Part 2. The techniques from [8] can be used to prove Part 1 as well, but in the present paper we give a shorter, more intuitive proof. The last part of Theorem 0.6 seems to be a new result, even for classical commutant lifting. Part 3 and the fact that a contractive interpolant $B$ with $\|B\|=\|A\|$ always exists imply the following corollary.

Corollary 0.7 Let $\left\{A, T^{\prime}, U^{\prime}, R, Q\right\}$ be a lifting data set with $\|A\|<1$. Then $\operatorname{Ker} D_{B_{V}}=\{0\}$ for each $V \in \mathbf{S}_{0}\left(\mathcal{G}, \mathcal{D}_{\omega^{*}}\right)$, i.e., for any $h \in \mathcal{H}$ and $V \in \mathbf{S}_{0}\left(\mathcal{G}, \mathcal{D}_{\omega^{*}}\right)$ we have $\left\|B_{V} h\right\|<\|h\|$.

The paper consists of six sections, not counting the present introduction. We start with a section where some preliminary system theory results that will be used throughout the paper is presented. In Sect. 2 we prove Proposition 0.3 and Theorem 0.2. The following section contains a proof of Theorem 0.5 restricted to the case that the contractive interpolant in question is the central contractive interpolant. We then proceed with an intermezzo about general Redheffer representations in Sect. 4. Finally, Theorems 0.5 and 0.6 are proved in Sects. 5 and 6, respectively. The order in the proofs goes in the reversed direction: we first prove analogous results on the level of Redheffer representations before proving Theorems 0.5 and 0.6 .

\section{System Theory Preliminaries}

In this section we review some results from linear system theory that will be useful in the sequel. See $[15,19]$ for a general overview. 
Contractive systems. A contractive system is a quadruple $\{X, B, C, D\}$ consisting of Hilbert space operators: $X$ on $\mathcal{X}, B$ from $\mathcal{U}$ to $\mathcal{X}, C$ from $\mathcal{X}$ to $\mathcal{Y}$ and $D$ mapping $\mathcal{U}$ into $\mathcal{Y}$ such that the system matrix

$$
\left[\begin{array}{ll}
X & B \\
C & D
\end{array}\right]:\left[\begin{array}{l}
\mathcal{X} \\
\mathcal{U}
\end{array}\right] \rightarrow\left[\begin{array}{l}
\mathcal{X} \\
\mathcal{Y}
\end{array}\right]
$$

is a contraction. Of particular interest will be the case when the system matrix is a co-isometry; we then say that $\{X, B, C, D\}$ is a co-isometric system. The system $\{X, B, C, D\}$ is said to be strongly stable in case the state operator $X$ is strongly stable, that is, if for each $x \in \mathcal{X}$ the sequence $X^{n} x$ converges to zero as $n \rightarrow \infty$. Since $X$ is contractive, we can define analytic functions $F$ and $W$ on $\mathbb{D}$ by

$$
F(\lambda)=D+\lambda C(I-\lambda X)^{-1} B \text { and } W(\lambda)=C(I-\lambda X)^{-1}(\lambda \in \mathbb{D}) .
$$

We refer to $F$ and $W$ as the transfer function and observability function associated with $\{X, B, C, D\}$, respectively. From the fact that the system matrix (1.1) is a co-isometry it follows that $F \in \mathbf{S}(\mathcal{U}, \mathcal{Y})$ and $W \in \mathbf{H}_{\text {ball }}^{2}(\mathcal{X}, \mathcal{Y})$. Hence $F$ defines a contractive multiplication operator $M_{F}$ from $H^{2}(\mathcal{U})$ to $H^{2}(\mathcal{Y})$, and $W$ defines a contraction $\Gamma_{W}$ from $\mathcal{X}$ into $H^{2}(\mathcal{Y})$ via $(0.1)$. The system $\{X, B, C, D\}$ is said to be observable in case $\operatorname{Ker} \Gamma_{W}=\{0\}$.

Two co-isometric systems $\left\{X_{1}, B_{1}, C_{1}, D_{1}\right\}$ and $\left\{X_{2}, B_{2}, C_{2}, D_{2}\right\}$ with the same input spaces $\mathcal{U}$ and output spaces $\mathcal{Y}$ but possibly different state spaces $\mathcal{X}_{1}$ respectively $\mathcal{X}_{2}$ are said to be unitarily equivalent if $D_{1}=D_{2}$ and there exists a unitary operator $\Theta$ mapping $\mathcal{X}_{1}$ onto $\mathcal{X}_{2}$ such that

$$
\Theta X_{1}=X_{2} \Theta, \quad C_{1}=C_{2} \Theta, \quad \Theta B_{1}=B_{2} .
$$

One easily verifies that unitarily equivalent systems have the same transfer function. The converse statement is true if the co-isometric systems are also observable.

Theorem 1.1 Let $\{X, B, C, D\}$ be a co-isometric system with transfer function $F \in \mathbf{S}(\mathcal{U}, \mathcal{Y})$ and observability function $W \in \mathbf{H}_{\text {ball }}^{2}(\mathcal{X}, \mathcal{Y})$. Then the operator

$$
\left[\begin{array}{ll}
M_{F} & \Gamma_{W}
\end{array}\right]:\left[\begin{array}{l}
H^{2}(\mathcal{U}) \\
\mathcal{X}
\end{array}\right] \rightarrow H^{2}(\mathcal{Y})
$$

is a co-isometry. Moreover, the operator (1.3) is unitary if and only if the system $\{X, B, C, D\}$ is strongly stable and the system matrix (1.1) is unitary.

Proof The statement for the case that (1.3) is unitary follows from Theorem III.10.4 in [13] and the fact that the positive operator $\Delta$ on $\mathcal{X}$ defined by

$$
\Delta^{2}=\text { strong- } \lim _{n \rightarrow \infty} X^{* n} X^{n}
$$

is zero if and only if $X$ is strongly stable. For the statement on co-isometric systems see Theorem 1.3 in [21].

The first part of Theorem 1.1 has the following converse result. 
Theorem 1.2 Let $F \in \mathbf{S}(\mathcal{U}, \mathcal{Y})$ and $W \in \mathbf{H}^{2}(\mathcal{X}, \mathcal{Y})$ be functions such that the operator matrix (1.3) is a co-isometry. Then there is a co-isometric system $\{X, B, C, D\}$ so that $F$ and $W$ are the associated transfer and observability functions.

Proof It follows from [1] that the function $F$ is the transfer function of an observable co-isometric system $\{\widetilde{X}, \widetilde{B}, \widetilde{C}, \widetilde{D}\}$, say with state space $\widetilde{\mathcal{X}}$. Let $\widetilde{W}$ be the associated observability function. From Theorem 1.1 we know that the operator matrix $\left[M_{F} \Gamma_{\tilde{W}}\right]$ is a co-isometry. Hence

$$
M_{F} M_{F^{*}}+\Gamma_{\widetilde{W}} \Gamma_{\widetilde{W}}^{*}=I=M_{F} M_{F^{*}}+\Gamma_{W} \Gamma_{W}^{*}, \quad \text { and thus } \quad \Gamma_{\widetilde{W}} \Gamma_{\widetilde{W}}^{*}=\Gamma_{W} \Gamma_{W}^{*} .
$$

By Douglas factorization lemma [10] there exists a unitary operator $\Lambda$ mapping $\overline{\operatorname{Im} \Gamma_{\widetilde{W}}^{*}}=\widetilde{\mathcal{X}}$ onto $\mathcal{N}=\overline{\operatorname{Im} \Gamma_{W}^{*}}$ defined by the identity $\Gamma_{\widetilde{W}} \Lambda^{*}=\Gamma_{W}$. Now set $\mathcal{M}=\mathcal{X} \ominus \mathcal{N}$ and define $\{X, B, C, D\}$ to be the co-isometric system given by the system matrix

$$
\left[\begin{array}{ll}
X & B \\
C & D
\end{array}\right]=\left[\begin{array}{ll|l}
\Lambda & 0 & 0 \\
0 & I_{\mathcal{M}} & 0 \\
\hline 0 & 0 & I_{\mathcal{Y}}
\end{array}\right]\left[\begin{array}{ll|l}
\widetilde{X} & 0 & \widetilde{B} \\
0 & I_{\mathcal{M}} & 0 \\
\hline \widetilde{C} & 0 & \widetilde{D}
\end{array}\right]\left[\begin{array}{ll|l}
\Lambda^{*} & 0 & 0 \\
0 & I_{\mathcal{M}} & 0 \\
\hline 0 & 0 & I_{\mathcal{Y}}
\end{array}\right] .
$$

Then $\{X, B, C, D\}$ and $\{\widetilde{X}, \widetilde{B}, \widetilde{C}, \widetilde{D}\}$ are unitarily equivalent, and thus $F$ is also the transfer function of $\{X, B, C, D\}$. Moreover, the observability function $\widehat{W}$ of $\{X, B, C, D\}$ satisfies $\widehat{W}(\lambda)=\left[\widetilde{W}(\lambda) \Lambda^{*} 0\right]$ for each $\lambda \in \mathbb{D}$. The latter implies that

$$
\Gamma_{\widehat{W}}=\left[\begin{array}{cc}
\Gamma_{\widetilde{W}} \Lambda^{*} & 0
\end{array}\right]=\Gamma_{W} .
$$

But then $\widehat{W}=W$, which proves our claim.

Redheffer cascading systems. Suppose we are given Hilbert spaces $\mathcal{U}_{1}, \mathcal{U}_{2}, \mathcal{Y}_{1}, \mathcal{Y}_{2}$, $\mathcal{X}$ and $\mathcal{X}^{\prime}$ and two operators $M_{1}$ mapping $\mathcal{X} \oplus \mathcal{U}_{1}$ into $\mathcal{X}^{\prime} \oplus \mathcal{Y}_{1}$ and $M_{2}$ mapping $\mathcal{X}^{\prime} \oplus \mathcal{U}_{2}$ into $\mathcal{X} \oplus \mathcal{Y}_{2}$ with operator matrix decompositions

$$
M_{1}=\left[\begin{array}{ll}
X_{1} & B_{1} \\
C_{1} & D_{1}
\end{array}\right]:\left[\begin{array}{l}
\mathcal{X} \\
\mathcal{U}_{1}
\end{array}\right] \rightarrow\left[\begin{array}{l}
\mathcal{X}^{\prime} \\
\mathcal{Y}_{1}
\end{array}\right], \quad M_{2}=\left[\begin{array}{ll}
X_{2} & B_{2} \\
C_{2} & D_{2}
\end{array}\right]:\left[\begin{array}{l}
\mathcal{X}^{\prime} \\
\mathcal{U}_{2}
\end{array}\right] \rightarrow\left[\begin{array}{l}
\mathcal{X} \\
\mathcal{Y}_{2}
\end{array}\right]
$$

Furthermore, assume that $I_{\mathcal{X}}-X_{2} X_{1}$ is invertible. Then $I_{\mathcal{X}^{\prime}}-X_{1} X_{2}$ is invertible, and we can form the Redheffer product $M_{1} \circ M_{2}$ of $M_{1}$ and $M_{2}$, which is the operator from $\mathcal{U}_{1} \oplus \mathcal{U}_{2}$ into $\mathcal{Y}_{1} \oplus \mathcal{Y}_{2}$ given by the operator matrix decomposition

$$
\left[\begin{array}{ll}
D_{1}+C_{1} X_{2}\left(I_{\mathcal{X}^{\prime}}-X_{1} X_{2}\right)^{-1} B_{1} & C_{1}\left(I_{\mathcal{X}}-X_{2} X_{1}\right)^{-1} B_{2} \\
C_{1}\left(I_{\mathcal{X}^{\prime}}-X_{1} X_{2}\right)^{-1} B_{1} & D_{2}+C_{2} X_{1}\left(I_{\mathcal{X}}-X_{2} X_{1}\right)^{-1} B_{2}
\end{array}\right] .
$$

For an elaborate discussion on Redheffer products we refer to [12, Chapter XIV]. The next proposition shows how norm properties of $M_{1}$ and $M_{2}$ carry over to $M_{1} \circ M_{2}$. 
Proposition 1.3 In case the block operator matrices $M_{1}$ and $M_{2}$ in (1.4) are both contractive, isometric, co-isometric or unitary, and $I-X_{2} X_{1}$ is invertible, the Redheffer product $M_{1} \circ M_{2}$ is also contractive, isometric, co-isometric or unitary, respectively.

Proof Since we assume $I_{\mathcal{X}}-X_{2} X_{1}$ to be invertible, the case where $M_{1}$ and $M_{2}$ are both contractive follows from Lemma XIV.1.2 in [12]. The case where $M_{1}$ and $M_{2}$ are isometries follows from Lemma XIV.1.2 in [12] as well, using identity (1.15) on Page 433 of [12]. The co-isometric case is obtained by applying the statement for the isometric case to $M_{1}^{*}$ and $M_{2}^{*}$, and finally the unitary case follows from the result when $M_{1}$ and $M_{2}$ are both isometric and co-isometric.

\section{Proofs of Theorem 0.2 and Proposition 0.3}

We start this section with a proof of Theorem 0.2 , followed by a proof of Proposition 0.3 .

Proof of Theorem 0.2 Assume that $\mathcal{U}, \mathcal{Y}, \mathcal{E}$ and $\mathcal{E}^{\prime}$ are Hilbert spaces, and

$$
\Psi_{1,1} \in \mathbf{S}\left(\mathcal{E}^{\prime}, \mathcal{E}\right), \quad \Psi_{1,2} \in \mathbf{H}_{\text {ball }}^{2}(\mathcal{U}, \mathcal{E}), \quad \Psi_{2,1} \in \mathbf{S}\left(\mathcal{E}^{\prime}, \mathcal{Y}\right), \quad \Psi_{2,2} \in \mathbf{H}_{\text {ball }}^{2}(\mathcal{U}, \mathcal{Y}),
$$

with $\Psi_{1,1}(0)=0$ such that the coefficient matrix (0.4) is a co-isometry. Set

$$
F(\lambda)=\left[\begin{array}{l}
\Psi_{1,1}(\lambda) \\
\Psi_{2,1}(\lambda)
\end{array}\right] \in \mathbf{S}\left(\mathcal{E}^{\prime}, \mathcal{E} \oplus \mathcal{Y}\right) \quad \text { and } \quad W(\lambda)=\left[\begin{array}{l}
\Psi_{1,2}(\lambda) \\
\Psi_{2,2}(\lambda)
\end{array}\right] \in \mathbf{H}_{\text {ball }}^{2}(\mathcal{U}, \mathcal{E} \oplus \mathcal{Y})
$$

Then $\left[\begin{array}{ll}M_{F} & \Gamma_{W}\end{array}\right]$ is a co-isometry from $H^{2}\left(\mathcal{E}^{\prime}\right) \oplus \mathcal{U}$ into $H^{2}(\mathcal{E} \oplus \mathcal{Y})$. According to Theorem 1.2 there exists a co-isometric system $\left\{X, B,\left[\begin{array}{c}C_{1} \\ C_{2}\end{array}\right],\left[\begin{array}{l}D_{1} \\ D_{2}\end{array}\right]\right\}$ with system matrix

$$
N=\left[\begin{array}{ll}
X & B \\
C_{1} & D_{1} \\
C_{2} & D_{2}
\end{array}\right]:\left[\begin{array}{l}
\mathcal{U} \\
\mathcal{E}^{\prime}
\end{array}\right] \rightarrow\left[\begin{array}{l}
\mathcal{U} \\
\mathcal{E} \\
\mathcal{Y}
\end{array}\right]
$$

such that $F$ and $W$ are the corresponding transfer function and observability function, respectively. The fact that $\Psi_{1,1}(0)=0$ implies that $D_{1}=0$, and thus, since the system matrix $N$ is a co-isometry, that $C_{1}=\Psi_{1,2}(0)$ is a co-isometry. Set $\mathcal{F}=\operatorname{Ker} C_{1}$ and $\mathcal{G}=\mathcal{U} \ominus \mathcal{F}$. Then $C_{1}$ maps $\mathcal{G}$ isometrically onto $\mathcal{E}^{\prime}$. Define $\omega_{1}=C_{2} \mid \mathcal{F}$ and $\omega_{2}=\left.X\right|_{\mathcal{F}}$, i.e.,

$$
N=\left[\begin{array}{ll}
\omega_{2} \Pi_{\mathcal{F}} & B \\
C_{1} P_{\mathcal{G}} & 0 \\
\omega_{1} \Pi_{\mathcal{F}} & D_{1}
\end{array}\right]
$$


By a special case of Parrot's lemma [27], again using that $N$ is a co-isometry, there exists a co-isometry $\varphi$ mapping $\mathcal{E}^{\prime}$ onto $\mathcal{D}_{\omega^{*}}$ such that

$$
\left[\begin{array}{l}
B \\
D_{1}
\end{array}\right]=D_{\omega^{*}} \varphi, \quad \text { where } \quad \omega=\left[\begin{array}{l}
\omega_{1} \\
\omega_{2}
\end{array}\right]: \mathcal{F} \rightarrow\left[\begin{array}{l}
\mathcal{Y} \\
\mathcal{U}
\end{array}\right]
$$

Let $\psi$ be the unitary map from $\mathcal{G}$ onto $\mathcal{E}$ given by $\psi=\left.C_{1}\right|_{\mathcal{G}}$. Then

$$
\left[\begin{array}{lll}
I_{\mathcal{U}} & 0 & 0 \\
0 & \psi & 0 \\
0 & 0 & I_{\mathcal{Y}}
\end{array}\right] N\left[\begin{array}{ll}
I_{\mathcal{U}} & 0 \\
0 & \varphi^{*}
\end{array}\right]=\left[\begin{array}{ll}
\omega_{2} \Pi_{\mathcal{F}} & \Pi_{\mathcal{U}} D_{\omega^{*}} \\
\Pi_{\mathcal{G}} & 0 \\
\omega_{1} \Pi_{\mathcal{F}} & \Pi_{\mathcal{Y}} D_{\omega^{*}}
\end{array}\right]
$$

Thus $\left\{X, B,\left[\begin{array}{l}C_{1} \\ C_{2}\end{array}\right],\left[\begin{array}{l}D_{1} \\ D_{2}\end{array}\right]\right\}$ is unitarily equivalent to the system $\{\widehat{X}, \widehat{B}, \widehat{C}, \widehat{D}\}$ given by

$$
\left[\begin{array}{ll}
\widehat{X} & \widehat{B} \\
\widehat{C} & \widehat{D}
\end{array}\right]=\left[\begin{array}{l|l}
\omega_{2} \Pi_{\mathcal{F}} & \Pi_{\mathcal{U}} D_{\omega^{*}} \\
\hline \Pi_{\mathcal{G}} & 0 \\
\omega_{1} \Pi_{\mathcal{F}} & \Pi_{\mathcal{Y}} D_{\omega^{*}}
\end{array}\right]:\left[\begin{array}{l}
\mathcal{U} \\
\mathcal{D}_{\omega^{*}}
\end{array}\right] \rightarrow\left[\begin{array}{l}
\mathcal{U} \\
\mathcal{G} \\
\mathcal{Y}
\end{array}\right]
$$

One easily verifies that

$$
\left[\begin{array}{l}
\Phi_{1,1}(\lambda) \\
\Phi_{2,1}(\lambda)
\end{array}\right]=\widehat{D}+\lambda \widehat{C}(I-\lambda \widehat{X})^{-1} \widehat{B} \quad \text { and } \quad\left[\begin{array}{l}
\Phi_{1,2}(\lambda) \\
\Phi_{2,2}(\lambda)
\end{array}\right]=\widehat{C}(I-\lambda \widehat{X})^{-1}
$$

where $\Phi_{1,1}$ and $\Phi_{2,1}$ are defined by (0.8) with $\omega$ as in (2.1). A straightforward computation shows that the transfer function $\widehat{F}$ and observability function $\widehat{W}$ of the co-isometric system $\{\widehat{X}, \widehat{B}, \widehat{C}, \widehat{D}\}$ given in (2.2) relate to $F$ and $W$ via

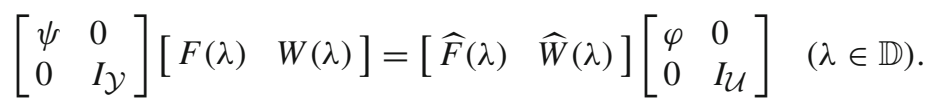

Thus, to prove Theorem 0.2 it remain to show that the contraction $\omega$ appear as the underlying contraction of a lifting data set. However, it is known from [18] that any contraction $\omega$ of the form in (2.1) with $\mathcal{F} \subset \mathcal{U}$, is the underlying contraction of some lifting data set. In fact, one easily verifies that the operators

$$
A=\left[\begin{array}{ll}
I \mathcal{Y} & 0
\end{array}\right]:\left[\begin{array}{l}
\mathcal{Y} \\
\mathcal{U}
\end{array}\right] \rightarrow \mathcal{Y}, T^{\prime}=0 \quad \text { on } \mathcal{Y}, R=\omega, Q=\Pi_{\mathcal{F}}^{*}: \mathcal{F} \rightarrow \mathcal{Y} \oplus \mathcal{U}
$$

define a lifting data set $\left\{A, T^{\prime}, U^{\prime}, R, Q\right\}$, with $U^{\prime}$ the Sz.-Nagy-Schäffer isometric lifting of $T^{\prime}$, which has $\omega$ as its underlying contraction.

To complete the proof, assume that the coefficient matrix $K_{0}$ in $(0.4)$ is unitary. Then $\left.K_{0}\right|_{H^{2}\left(\mathcal{E}^{\prime}\right)}$ is an isometry, and thus, in particular, for each $e \in \mathcal{E}^{\prime}$ there exists a $\lambda \in \mathbb{D}$ such that $\Psi_{1,1}(\lambda) e \neq 0$ or $\Psi_{2,1}(\lambda) e \neq 0$. So, by $(0.13)$ and the fact that $\psi$ is unitary, $\varphi e \neq 0$ for all $e \in \mathcal{E}^{\prime}$, and thus $\varphi$ is, in fact, unitary. 
Proof of Proposition 0.3 Let $\omega$ and $\omega^{\prime}$ denote the underlying contractions of the data sets $\left\{A, T^{\prime}, U^{\prime}, R, Q\right\}$ and $\left\{\widetilde{A}, \widetilde{T}^{\prime}, \widetilde{U}^{\prime}, \widetilde{R}, \widetilde{Q}\right\}$, respectively.

Since the coefficient matrix (0.4) is assumed to be unitary, we may without loss of generality assume that the Redheffer coefficients defined by $\omega$ and $\omega^{\prime}$ via (0.8) are equal to the given Redheffer coefficients (0.2). Indeed, this is the case because, according to the last part of Theorem 0.2 , the three sets of Redheffer coefficients are the same up to unitary transformations between $\mathcal{E}$ and $\mathcal{G}=\mathcal{U} \ominus \mathcal{F}$ and between $\mathcal{E}^{\prime}$ and $\mathcal{D}_{\omega^{*}}$ and $\mathcal{D}_{\omega^{*}}$, and these unitary transformations do not affect whether $\omega$ and $\omega^{\prime}$ are unitarily equivalent, or not.

Now let $\{\widehat{X}, \widehat{B}, \widehat{C}, \widehat{D}\}$ be the co-isometric system given by (2.2) and define $\left\{\widehat{X}^{\prime}, \widehat{B}^{\prime}, \widehat{C}^{\prime}, \widehat{D}^{\prime}\right\}$ accordingly with $\omega$ replaced by $\omega^{\prime}$. Then both systems have the same transfer function $F$ and observability function $W$ that are given by

$$
F(\lambda)=\left[\begin{array}{l}
\Psi_{1,1}(\lambda) \\
\Psi_{2,1}(\lambda)
\end{array}\right] \text { and } W(\lambda)=\left[\begin{array}{l}
\Psi_{1,2}(\lambda) \\
\Psi_{2,2}(\lambda)
\end{array}\right]
$$

By assumption, the coefficient matrix is unitary. This implies that the operator $\Gamma_{W}$, defined via (0.1), is an isometry. In particular, $\operatorname{Ker} \Gamma_{W}=\{0\}$. Hence the two co-isometric systems $\{\widehat{X}, \widehat{B}, \widehat{C}, \widehat{D}\}$ and $\left\{\widehat{X}^{\prime}, \widehat{B}^{\prime}, \widehat{C}^{\prime}, \widehat{D}^{\prime}\right\}$ are observable and have the same transfer function. This implies that they are unitarily equivalent. It then follows from the definition of the systems $\{\widehat{X}, \widehat{B}, \widehat{C}, \widehat{D}\}$ and $\left\{\widehat{X}^{\prime}, \widehat{B}^{\prime}, \widehat{C}^{\prime}, \widehat{D}^{\prime}\right\}$ that the operator $\Theta$ on $\mathcal{U}$ that establishes this unitary equivalence is also the operator that establishes the unitary equivalence of $\omega$ and $\omega^{\prime}$.

\section{Proof of Theorem 0.5 for the Central Solution}

Let $B_{c}$ denote the central contractive interpolant, that is, $B_{c}=B_{0}$, where $\underline{0}$ denotes the constant Schur class function whose value is the zero operator in $\mathscr{L}\left(\mathcal{G}, \mathcal{D}_{\omega^{*}}\right)$. In this section we prove Theorem 0.5 restricted to the case that the contractive interpolant $B$ is equal to $B_{c}$. To be more precise, we prove the following proposition.

Proposition 3.1 Let $\left\{A, T^{\prime}, U^{\prime}, R, Q\right\}$ be a lifting data set such that the underlying contraction $\omega$ is an isometry and $\omega_{2} \Pi_{\mathcal{F}}$ on $\mathcal{D}_{A}$ is strongly stable. Then the set $\mathscr{V}_{B_{c}}$ in (0.15) consists of just one element if and only if $\Gamma_{\Phi_{1,2}} \mathcal{D}_{A}$ is dense in $H^{2}(\mathcal{G})$.

In order to prove Proposition 3.1 it is convenient to first prove the next lemma. Recall that $E_{\mathcal{G}} \in \mathscr{L}\left(\mathcal{G}, H^{2}(\mathcal{G})\right)$ stands for the canonical embedding of $\mathcal{G}$ into the set of constant functions in $H^{2}(\mathcal{G})$.

Lemma 3.2 Let $\mathcal{K} \subset H^{2}(\mathcal{G})$ be an invariant subspace of the backward shift $S_{\mathcal{G}}^{*}$. Then $\mathcal{G}_{0}:=E_{\mathcal{G}} \mathcal{G} \subset \mathcal{K}$ and $S_{\mathcal{G}}^{*} \mathcal{K}=\mathcal{K}$ if and only if $\mathcal{K}=H^{2}(\mathcal{G})$.

Proof If $\mathcal{K}=H^{2}(\mathcal{G})$, then obviously $\mathcal{G}_{0} \subset \mathcal{K}$ and $S_{\mathcal{G}}^{*} \mathcal{K}=\mathcal{K}$. Conversely, assume that $\mathcal{G}_{0} \subset \mathcal{K}$ and $S_{\mathcal{G}}^{*} \mathcal{K}=\mathcal{K}$. Then $S_{\mathcal{G}}^{n} \mathcal{G}_{0} \subset S_{\mathcal{G}}^{n} \mathcal{K}=S_{\mathcal{G}}^{n} S_{\mathcal{G}}^{* n} \mathcal{K}$ for $n=0,1,2, \ldots$. Since $\mathcal{G}_{0}=S_{\mathcal{G}}^{0} \mathcal{G}_{0} \subset \mathcal{K}$, we obtain that $S_{\mathcal{G}} \mathcal{G}_{0} \subset S_{\mathcal{G}} S_{\mathcal{G}}^{*} \mathcal{K}=\mathcal{K} \ominus \mathcal{G}_{0} \subset \mathcal{K}$, and with a similar 
argument it follows, recursively, that

$$
S_{\mathcal{G}}^{n} \mathcal{G}_{0} \subset S_{\mathcal{G}}^{n} S_{\mathcal{G}}^{* n} \mathcal{K}=\mathcal{K} \ominus\left(\oplus_{k=0}^{n-1} S_{\mathcal{G}}^{k} \mathcal{G}_{0}\right) \subset \mathcal{K}
$$

for each nonnegative integer $n$. The latter inclusions imply that $\mathcal{K}=H^{2}(\mathcal{G})$.

Proof of Proposition 3.1 Note that $B_{c}$ is of the form (0.11) with $H_{V}$ equal to $H_{c}:=$ $\Phi_{2,2}$. Let $\omega_{B_{c}}$ be the contraction defined according to (0.16). It follows from the last statement of Theorem 0.1 and the remark in the sentence after $(0.16)$ that $\omega_{B_{c}}$ is an isometry. So, by Proposition 0.4 , it suffices to prove that $\Gamma_{\Phi_{1,2}} \mathcal{D}_{A}$ is dense in $H^{2}(\mathcal{G})$ if and only if $\mathcal{F}_{B_{c}}=\mathcal{D}_{\Gamma_{\Phi_{2,2}}}$ or $\omega_{B_{c}} \mathcal{F}_{B_{c}}=\mathcal{D}_{\Gamma_{\Phi_{2,2}}}$.

Since, by Theorem 0.1 , the coefficient matrix $K_{0}$ is unitary we have

$$
D_{\Gamma_{H_{C}}}^{2}=D_{\Gamma_{\Phi_{2,2}}}^{2}=I-\Gamma_{\Phi_{2,2}}^{*} \Gamma_{\Phi_{2,2}}=\Gamma_{\Phi_{1,2}}^{*} \Gamma_{\Phi_{1,2}} .
$$

Hence there exists a unique unitary operator $\rho$ mapping $\mathcal{D}_{H_{c}}$ onto $\mathcal{K}:=\overline{\Gamma_{\Phi_{1,2}} \mathcal{D}_{A}}$ defined by $\rho D_{\Gamma_{\Phi_{2}, 2}}=\Gamma_{\Phi_{1,2}}$. The fact that $\Gamma_{\Phi_{1,2}}$ is contractive and $\Phi_{1,2}(0)=\Pi_{\mathcal{G}}$ implies that $\mathcal{G}_{0}:=E_{\mathcal{G}} \mathcal{G} \subset \mathcal{K}$ and that $\rho$ maps $\mathcal{F}_{B_{c}}=D_{\Gamma_{\Phi_{2}, 2}} \mathcal{F}$ onto $\widetilde{\mathcal{F}}_{B_{c}}:=\mathcal{K} \ominus \mathcal{G}_{0}$. In particular, $\mathcal{F}_{B_{c}}=\mathcal{D}_{\Gamma_{\Psi_{2,2}}}$ holds if and only if $\mathcal{G}=\{0\}$, from which the denseness of $\Gamma_{\Phi_{1,2}} \mathcal{D}_{A}$ in $H^{2}(\mathcal{G})$ obviously follows.

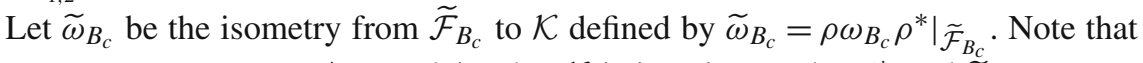
$\widetilde{\omega}_{B_{c}} \Pi_{\widetilde{\mathcal{F}}_{B_{C}}}=\rho \omega_{B_{c}} \Pi_{\mathcal{F}_{B_{C}}} \rho^{*}$. We claim that $\mathcal{K}$ is invariant under $S_{\mathcal{G}}^{*}$ and $\widetilde{\omega}_{B_{c}} \Pi_{\mathcal{F}_{B_{C}}}=$ $S_{\mathcal{G}}^{*} \mid \mathcal{K}$. The latter, which implies the former, follows since

$$
\begin{aligned}
\widetilde{\omega}_{B_{c}} \Pi_{\tilde{\mathcal{F}}_{B_{C}}} \Gamma_{\Phi_{1,2}} & =\rho \omega_{B_{c}} \Pi_{\mathcal{F}_{B_{c}}} \rho^{*} \Gamma_{\Phi_{1,2}}=\rho \omega_{B_{c}} \Pi_{\mathcal{F}_{B_{c}}} D_{\Gamma_{\Phi_{2,2}}}=\rho \omega_{B_{c}} D_{\Gamma_{\Phi_{2,2}}} P_{\mathcal{F}} \\
& =\rho D_{\Gamma_{\Phi_{2,2}}} \omega_{2} \Pi_{\mathcal{F}}=\Gamma_{\Phi_{1,2}} \omega_{2} \Pi_{\mathcal{F}}=S_{\mathcal{G}}^{*} \Gamma_{\Phi_{1,2}} P_{\mathcal{F}}=S_{\mathcal{G}}^{*} \Gamma_{\Phi_{1,2}} .
\end{aligned}
$$

The third identity follows from the fact that $\left.\Gamma_{\Phi_{2,2}}\right|_{\mathcal{G}}=0$, and thus $D_{\Gamma_{\Phi_{2,2}}} \mathcal{G}=\mathcal{G}$. For the last identity it is again used that $\Gamma_{\Phi_{1,2}}$ is a contraction and $\Phi_{1,1}(0) \stackrel{2,2}{=} \Pi_{\mathcal{G}}$, which implies that $\Gamma_{\Phi_{1,2}} \mathcal{G}=\mathcal{G}_{0}=\operatorname{Ker} S_{\mathcal{G}}^{*}$.

We have thus shown that $\mathcal{K}$ is a backward shift invariant subspace of $H^{2}(\mathcal{G})$ with $\mathcal{G}_{0} \subset \mathcal{K}$. It then follows from Lemma 3.2 that $\mathcal{K}=H^{2}(\mathcal{G})$ if and only if $\widetilde{\omega}_{B_{c}} \widetilde{\mathcal{F}}_{B_{c}}=$ $S_{\mathcal{G}}^{*} \mathcal{K}=\mathcal{K}$. This is the same as saying that $\Gamma_{\Phi_{1,2}} \mathcal{D}_{A}$ is dense in $H^{2}(\mathcal{G})$ if and only if $\omega_{B_{c}} \mathcal{F}_{B_{c}}=\mathcal{D}_{\Gamma_{\Phi_{2,2}}}$.

As a result of Theorem 0.2 we obtain the following analogue of Proposition 3.1 in the setting of general Redheffer representations.

Proposition 3.3 Let $\mathscr{R}_{\Psi}$ be a Redheffer representation with Redheffer coefficients $\Psi_{1,1}, \Psi_{1,2}, \Psi_{2,1}$ and $\Psi_{2,2}$ as in (0.2) such that the coefficient matrix (0.4) is unitary. Then the set

$$
\mathscr{W}_{H_{\underline{0}}}:=\left\{V \in \mathbf{S}\left(\mathcal{E}, \mathcal{E}^{\prime}\right) \mid H_{\underline{0}}=H_{V}\right\}
$$

is equal to $\{\underline{0}\}$ if and only if $\Gamma_{\Psi_{1,2}} \mathcal{U}$ is dense in $H^{2}(\mathcal{E})$. 
Proof The result follows immediately from Theorem 0.2 and Proposition 3.1.

\section{Intermezzo: Redheffer Representations}

Assume we are given Hilbert spaces $\mathcal{U}, \mathcal{Y}, \mathcal{E}$ and $\mathcal{E}^{\prime}$ and operator-valued functions

$$
\Psi_{1,1} \in \mathbf{S}\left(\mathcal{E}^{\prime}, \mathcal{E}\right), \quad \Psi_{1,2} \in \mathbf{H}_{\text {ball }}^{2}(\mathcal{U}, \mathcal{E}), \quad \Psi_{2,1} \in \mathbf{S}\left(\mathcal{E}^{\prime}, \mathcal{Y}\right), \quad \Psi_{2,2} \in \mathbf{H}_{\text {ball }}^{2}(\mathcal{U}, \mathcal{Y})
$$

with $\Psi_{1,1}(0)=0$. In the present section we derive some results for the Redheffer representation $\mathscr{R}_{\Psi}$ with coefficients $\Psi_{1,1}, \Psi_{1,2}, \Psi_{2,1}$ and $\Psi_{2,2}$.

In case the coefficient matrix

$$
K_{0}=\left[\begin{array}{ll}
M_{\Psi_{1,1}} & \Gamma_{\Psi_{1,2}} \\
M_{\Psi_{2,1}} & \Gamma_{\Psi_{2,2}}
\end{array}\right]\left[\begin{array}{l}
H^{2}\left(\mathcal{E}^{\prime}\right) \\
\mathcal{U}
\end{array}\right] \rightarrow\left[\begin{array}{l}
H^{2}(\mathcal{E}) \\
H^{2}(\mathcal{Y})
\end{array}\right]
$$

is a co-isometry, it follows from Theorems 0.2 and 0.1 that $\mathscr{R}_{\Psi}$ maps $\mathbf{S}\left(\mathcal{E}, \mathcal{E}^{\prime}\right)$ into $\mathbf{H}_{\text {ball }}^{2}(\mathcal{U}, \mathcal{Y})$. The next proposition shows that this is still true if the coefficient matrix is a contraction.

Proposition 4.1 Let $\Psi_{1,1}, \Psi_{1,2}, \Psi_{2,1}$ and $\Psi_{2,2}$ be as in (4.1) with $\Psi_{1,1}(0)=0$ and assume that (4.2) is contractive. Then $\mathscr{R}_{\Psi}$ maps $\mathbf{S}\left(\mathcal{E}, \mathcal{E}^{\prime}\right)$ into $\mathbf{H}_{\text {ball }}^{2}(\mathcal{U}, \mathcal{Y})$.

Proof For the case that the coefficient matrix $K_{0}$ is a co-isometry, the statement follows right away from Theorems 0.1 and 0.2. Now assume that $K_{0}$ is contractive. By Parrot's lemma [27] there exist a Hilbert space $\mathcal{V}$ and functions $\Psi_{1,3} \in \mathbf{H}_{\text {ball }}^{2}(\mathcal{V}, \mathcal{E})$ and $\Psi_{2,3} \in \mathbf{H}_{\text {ball }}^{2}(\mathcal{V}, \mathcal{Y})$ such that

$$
\widetilde{K}_{0}=\left[\begin{array}{lll}
M_{\Psi_{1,1}} & \Gamma_{\Psi_{1,2}} & \Gamma_{\Psi_{1,3}} \\
M_{\Psi_{2,1}} & \Gamma_{\Psi_{2,2}} & \Gamma_{\Psi_{2,3}}
\end{array}\right]:\left[\begin{array}{l}
H^{2}\left(\mathcal{E}^{\prime}\right) \\
\mathcal{U} \\
\mathcal{V}
\end{array}\right] \rightarrow\left[\begin{array}{l}
H^{2}(\mathcal{E}) \\
H^{2}(\mathcal{Y})
\end{array}\right]
$$

is a co-isometry. Set $\widetilde{\Psi}_{1,2}(\lambda)=\left[\Psi_{1,2}(\lambda) \quad \Psi_{1,3}(\lambda)\right] \in \mathbf{H}_{\text {ball }}^{2}(\mathcal{U} \oplus \mathcal{V}, \mathcal{E})$ and $\widetilde{\Psi}_{2,2}(\lambda)=$ $\left[\Psi_{2,2}(\lambda) \quad \Psi_{2,3}(\lambda)\right] \in \mathbf{H}_{\text {ball }}^{2}(\mathcal{U} \oplus \mathcal{V}, \mathcal{Y})$. It follows that the Redheffer representation $\mathscr{R}_{\widetilde{\Psi}}$ with Redheffer coefficients $\Psi_{1,1}, \widetilde{\Psi}_{1,2}, \Psi_{2,1}$ and $\widetilde{\Psi}_{2,2}$ has a co-isometric coefficient matrix, and thus maps $\mathbf{S}\left(\mathcal{E}, \mathcal{E}^{\prime}\right)$ into $\mathbf{H}_{\text {ball }}^{2}(\mathcal{U} \oplus \mathcal{V}, \mathcal{Y})$. The statement now follows because $\mathscr{R}_{\Psi}[V](\lambda)=\left.\mathscr{R}_{\widetilde{\Psi}}[V](\lambda)\right|_{\mathcal{U}}$ for each $\lambda \in \mathbb{D}$ and $V \in \mathbf{S}\left(\mathcal{E}, \mathcal{E}^{\prime}\right)$. Hence $H_{V} \in \mathbf{H}_{\text {ball }}^{2}(\mathcal{U}, \mathcal{Y})$.

As in the relaxed commutant lifting setting, the map $\mathscr{R}_{\Psi}$ is in general not one-to-one. This is the underlying obstruction preventing the obviously sufficient condition " $\mathcal{E}=$ $\{0\}$ or $\mathcal{E}^{\prime}=\{0\}$ " for $\mathscr{R}_{\Psi}\left[\mathbf{S}\left(\mathcal{E}, \mathcal{E}^{\prime}\right)\right]$ to be a singleton from also being necessary. Necessary and sufficient conditions under which the range of $\mathscr{R}_{\Psi}$ is a singleton, in the relaxed commutant lifting setting, were obtained in [25] for the case that $R^{*} R=Q^{*} Q$ and in [21] for the general case. An adaptation of the proof in [21] gives the following result. 
Proposition 4.2 Let $\Psi_{1,1}, \Psi_{1,2}, \Psi_{2,1}$ and $\Psi_{2,2}$ be as in (4.1) with $\Psi_{1,1}(0)=0$ and assume that the coefficient matrix (4.2) is contractive. Then the range of $\mathscr{R}_{\Psi}$ is a singleton if and only if $\Psi_{1,2}(\lambda)=0$ for each $\lambda \in \mathbb{D}$ or $\Psi_{2,1}(\lambda)=0$ for each $\lambda \in \mathbb{D}$. In case the coefficient matrix is a co-isometry, the range of $\mathscr{R}_{\Psi}$ is a singleton if and only if $\mathcal{E}=\{0\}$ or $\Gamma_{\Psi_{2,2}}$ is a co-isometry.

Proof The statement for the case that the coefficient matrix is a co-isometry follows from Theorem 0.2 above and Theorem 0.2 in [21]. The result for the case that the coefficient matrix is contractive can easily be deduced from the proof of Theorem 0.2 in [21].

We now restrict our attention to the Schur class functions in the open unit ball $\mathbf{S}_{0}\left(\mathcal{E}, \mathcal{E}^{\prime}\right)$ of $\mathbf{H}^{\infty}\left(\mathcal{E}, \mathcal{E}^{\prime}\right)$, i.e., $V \in \mathbf{H}^{\infty}\left(\mathcal{E}, \mathcal{E}^{\prime}\right)$ with $\|V\|_{\infty}<1$. For $V \in \mathbf{S}_{0}\left(\mathcal{E}, \mathcal{E}^{\prime}\right)$ the multiplication operator $M_{V}$ is a strict contraction, which enables us to express the operator $\Gamma_{H_{V}}$ associated with $H_{V}=\mathscr{R}_{\Psi}[V]$ via (0.1) explicitly as

$$
\Gamma_{H_{V}}=\Gamma_{\Psi_{2,2}}+M_{\Psi_{2,1}} M_{V}\left(I-M_{\Psi_{1,1}} M_{V}\right)^{-1} \Gamma_{\Psi_{1,2}} .
$$

We write $R_{V}$ for the rotation operator associated with the strict contraction $M_{V}$ :

$$
R_{V}:=\left[\begin{array}{ll}
M_{V} & D_{M_{V}^{*}} \\
-D_{M_{V}} & M_{V}^{*}
\end{array}\right]:\left[\begin{array}{l}
H^{2}(\mathcal{E}) \\
H^{2}\left(\mathcal{E}^{\prime}\right)
\end{array}\right] \rightarrow\left[\begin{array}{l}
H^{2}\left(\mathcal{E}^{\prime}\right) \\
H^{2}(\mathcal{E})
\end{array}\right] .
$$

Then $R_{V}$ is unitary, and, since $I-M_{\Psi_{1,1}} M_{V}$ is invertible, we can form the Redheffer product $K_{V}=R_{V} \circ K_{0}$ of $R_{V}$ with the coefficient matrix $K_{0}$ in (4.2), which gives us the following block operator matrix

$$
K_{V}=\left[\begin{array}{ll}
M_{V}^{*}+D_{M_{V}}\left(I-M_{\Psi_{1,1}} M_{V}\right)^{-1} M_{\Psi_{1,1}} & -D_{M_{V}}\left(I-M_{\Psi_{1,1}} M_{V}\right)^{-1} \Gamma_{\Psi_{1,2}} \\
M_{\Psi_{2,1}}\left(I-M_{V} M_{\Psi_{1,1}}\right)^{-1} D_{M_{V}^{*}} & \Gamma_{H_{V}}
\end{array}\right]
$$

that maps $H^{2}\left(\mathcal{E}^{\prime}\right) \oplus \mathcal{U}$ into $H^{2}(\mathcal{E}) \oplus H^{2}(\mathcal{Y})$. Note that in case $V=\underline{0}$, the constant function with value $0 \in \mathscr{L}\left(\mathcal{E}, \mathcal{E}^{\prime}\right), K_{V}$ is equal to the coefficient matrix $K_{0}$.

Proposition 4.3 For each $V \in \mathbf{S}_{0}\left(\mathcal{E}, \mathcal{E}^{\prime}\right)$ the operator matrix $K_{V}$ is contractive, co-isometric, isometric or unitary whenever the coefficient matrix $K_{0}$ in (4.2) is contractive, co-isometric, isometric or unitary, respectively.

Proof The result follows right away from Proposition 1.3 and the fact that the rotation operator $R_{V}$ is unitary.

\section{Proof of Theorem 0.5}

Before giving the proof of Theorem 0.5, we first prove the following Redheffer representation analogue. 
Theorem 5.1 Let $\Psi_{1,1}, \Psi_{1,2}, \Psi_{2,1}$ and $\Psi_{2,2}$ be as in (4.1) with $\Psi_{1,1}(0)=0$ and assume that the coefficient matrix $K_{0}$ in (4.2) is unitary. For each $V \in \mathbf{S}_{0}\left(\mathcal{E}, \mathcal{E}^{\prime}\right)$ the set

$$
\mathscr{W}_{H_{V}}=\left\{\tilde{V} \in \mathbf{S}\left(\mathcal{E}, \mathcal{E}^{\prime}\right) \mid H_{V}=H_{\tilde{V}}\right\}
$$

is equal to $\{V\}$ if and only if $\Gamma_{\Psi_{1,2}} \mathcal{U}$ is dense in $H^{2}(\mathcal{E})$. In particular, if $\mathscr{W}_{H_{V}}=\{V\}$ for some $V \in \mathbf{S}_{0}\left(\mathcal{E}, \mathcal{E}^{\prime}\right)$, then $\mathscr{W}_{H_{V}}=\{V\}$ for all $V \in \mathbf{S}_{0}\left(\mathcal{E}, \mathcal{E}^{\prime}\right)$.

Proof By Theorem 0.2 there exist a lifting data set $\left\{A, T^{\prime}, U^{\prime}, R, Q\right\}$ with underlying contraction $\omega$ as in (0.7) and associated Redheffer coefficients $\Phi_{1,1}, \Phi_{1,2}, \Phi_{2,1}$ and $\Phi_{2,2}$ given by (0.8), and unitary operators $\psi$ mapping $\mathcal{E}$ onto $\mathcal{G}=\mathcal{D}_{A} \ominus \mathcal{F}$ and $\varphi$ from $\mathcal{E}^{\prime}$ onto $\mathcal{D}_{\omega^{*}}$ such that $(0.13)$ holds. Thus $\Psi_{1,1}, \Psi_{1,2}, \Psi_{2,1}$ and $\Psi_{2,2}$ can be identified with coefficients $\Phi_{1,1}, \Phi_{1,2}, \Phi_{2,1}$ and $\Phi_{2,2}$, up to the two unitary maps $\psi$ and $\varphi$. In particular, the coefficient matrix (0.10) is unitary as well, which, according to Theorem 0.1 , implies that $\omega$ is an isometry.

Now fix a $V \in \mathbf{S}_{0}\left(\mathcal{E}, \mathcal{E}^{\prime}\right)$. The relation between the two sets of Redheffer coefficients and the lifting data set $\left\{A, T^{\prime}, U^{\prime}, R, Q\right\}$ implies that we can define a contraction $\omega_{H_{V}}$ by

$$
\omega_{H_{V}}: \mathcal{F}_{H_{V}}=\overline{D_{\Gamma_{H_{V}}} \mathcal{F}} \rightarrow \mathcal{D}_{\Gamma_{H_{V}}}, \quad \omega_{H_{V}} D_{\Gamma_{H_{V}}} \mid \mathcal{F}=D_{\Gamma_{H_{V}}} \omega_{2}
$$

In fact, $\omega_{H_{V}}$ is an isometry, because $\omega$ is an isometry; see the paragraph preceding Proposition 0.4. Moreover, by Proposition 0.4 and from the fact that $\omega_{H_{V}}$ is an isometry, it follows that $\mathscr{W}_{H_{V}}=\{V\}$ if and only if $\mathcal{F}_{H_{V}}=\mathcal{D}_{\Gamma_{H_{V}}}$ or $\omega_{H_{V}} \mathcal{F}_{H_{V}}=\mathcal{D}_{\Gamma_{H_{V}}}$.

Since the coefficient matrix (4.2) is unitary, we obtain from Proposition 4.3 that the operator $K_{V}$ in (4.5) is unitary. In particular,

$$
D_{\Gamma_{H_{V}}}^{2}=I-\Gamma_{H_{V}}^{*} \Gamma_{H_{V}}=C_{V}^{*} C_{V},
$$

where we define $C_{V}$ by

$$
C_{V}=Y_{V} \Gamma_{\Psi_{1,2}} \text { with } Y_{V}=D_{M_{V}}\left(I-M_{\Psi_{1,1}} M_{V}\right)^{-1} \text {. }
$$

Set $\mathcal{K}_{V}=\overline{C_{V} \mathcal{U}}$. According to Douglas' factorization lemma [10], the identity $\rho D_{\Gamma_{H_{V}}}=C_{V}$ defines a unitary map $\rho$ from $\mathcal{D}_{\Gamma_{H_{V}}}$ onto $\mathcal{K}_{V}$. Set

$$
\widetilde{\omega}_{H_{V}}=\left.\rho \omega_{H_{V}} \rho^{*}\right|_{\widetilde{\mathcal{F}}_{H_{V}}}, \quad \text { where } \widetilde{\mathcal{F}}_{H_{V}}=\rho \mathcal{F}_{H_{V}}
$$

Then $\widetilde{\omega}_{H_{V}}$ is an isometry from $\widetilde{\mathcal{F}}_{H_{V}}$ into $\mathcal{K}_{V}$, and $\mathscr{W}_{H_{V}}=\{V\}$ if and only if

$$
\widetilde{\mathcal{F}}_{H_{V}}=\overline{Y_{V} \Gamma_{\Psi_{1,2}} \mathcal{F}}=\mathcal{K}_{V} \quad \text { or } \quad \widetilde{\omega}_{H_{V}} \widetilde{\mathcal{F}}_{H_{V}}=\overline{Y_{V} \Gamma_{\Psi_{1,2}} \omega_{2} \mathcal{F}}=\mathcal{K}_{V}
$$


Since $\left\|M_{V}\right\|=\|V\|_{\infty}<1, D_{M_{V}}$ is invertible, and hence $Y_{V}$ is invertible. But then (5.2) holds if and only if

$$
\overline{\Gamma_{\Psi_{1,2}} \mathcal{F}}=\overline{\Gamma_{\Psi_{1,2}} \mathcal{U}} \text { or } \overline{\Gamma_{\Psi_{1,2}} \omega_{2} \mathcal{F}}=\overline{\Gamma \Psi_{1,2} \mathcal{U}}
$$

The conditions in (5.3) are equal to those in (5.2) in case $V=\underline{0}$, the constant function in $\mathbf{S}\left(\mathcal{E}, \mathcal{E}^{\prime}\right)$ whose value is the zero operator. This case was resolved in Proposition 3.3, from which we infer that $\mathscr{W}_{H_{V}}=\{V\}$ if and only if $\Gamma_{\Psi_{1,2}} \mathcal{U}$ is dense in $H^{2}(\mathcal{E})$.

Proof of Theorem 0.5 Let $\left\{A, T^{\prime}, U^{\prime}, R, Q\right\}$ be a lifting data set such that the underlying contraction $\omega$ is an isometry and $\omega_{2} \Pi_{\mathcal{F}}$ on $\mathcal{D}_{A}$ is strongly stable. Then the coefficient matrix (0.10) for the Redheffer coefficients (0.8) associated with the lifting data set $\left\{A, T^{\prime}, U^{\prime}, R, Q\right\}$ is unitary according to Theorem 0.1 .

By Parrott's lemma [27], the operators $B_{V}$ and $\Gamma_{V}$ in (0.11) determine each other uniquely, for any $V \in \mathbf{S}\left(\mathcal{D}_{A}, \mathcal{D}_{T^{\prime}}\right)$. This implies that $\mathscr{V}_{B_{V}}=\mathscr{W}_{H_{V}}$, where $\mathscr{V}_{B_{V}}$ and $\mathscr{W}_{H_{V}}$ are given by $(0.15)$ and (5.1), respectively. Therefore, we obtain Theorem 0.5 by applying Theorem 5.1 to the Redheffer representation $\mathscr{R}_{\Phi}$.

\section{Proof of Theorem 0.6}

In this section we derive Theorem 0.6 from the following harmonic maximal principle for Redheffer representations.

Theorem 6.1 Let $\Psi_{1,1}, \Psi_{1,2}, \Psi_{2,1}$ and $\Psi_{2,2}$ be as in (4.1) with $\Psi_{1,1}(0)=0$ and assume that the coefficient matrix (4.2) is contractive. Then, for the Redheffer representation $\mathscr{R}_{\Psi}$ we have:

(1) If there exists a $\widetilde{V} \in \mathbf{S}_{0}\left(\mathcal{E}, \mathcal{E}^{\prime}\right)$ with $\left\|\Gamma_{H_{\tilde{V}}}\right\|<1$, then $\left\|\Gamma_{H_{V}}\right\|<1$ for any $V \in \mathbf{S}_{0}\left(\mathcal{E}, \mathcal{E}^{\prime}\right)$.

(2) If there exists a $\widetilde{V} \in \mathbf{S}_{0}\left(\mathcal{E}, \mathcal{E}^{\prime}\right)$ with $\left\|\Gamma_{H_{\tilde{V}}}\right\|=1$, then $\left\|\Gamma_{H_{V}}\right\|=1$ for any $V \in \mathbf{S}_{0}\left(\mathcal{E}, \mathcal{E}^{\prime}\right)$.

(3) Moreover, for $V \in \mathbf{S}_{0}\left(\mathcal{E}, \mathcal{E}^{\prime}\right)$ and $\widetilde{V} \in \mathbf{S}\left(\mathcal{E}, \mathcal{E}^{\prime}\right)$ we have $\operatorname{Ker} D_{\Gamma_{H_{V}}} \subset \operatorname{Ker} D_{\Gamma_{H_{\tilde{V}}}}$. In particular, $\operatorname{Ker} D_{\Gamma_{H_{V}}}=\operatorname{Ker} D_{\Gamma_{H_{\tilde{V}}}}$ in case $\widetilde{V}, V \in \mathbf{S}_{0}\left(\mathcal{E}, \mathcal{E}^{\prime}\right)$.

Proof We first prove (3). Let $V \in \mathbf{S}_{0}\left(\mathcal{E}, \mathcal{E}^{\prime}\right)$ and $u \in \operatorname{Ker} D_{\Gamma_{H_{V}}}$, i.e., $\left\|\Gamma_{H_{V}} u\right\|=\|u\|$. Since the operator matrix $K_{V}$ of Proposition 4.3 is contractive, it follows (see the second column in (4.5)) that $-D_{M_{V}}\left(I-M_{\Psi_{1,1}} M_{V}\right)^{-1} \Gamma_{\Psi_{1,2}} u=0$. The operator $D_{M_{V}}\left(I-M_{\Psi_{1,1}} M_{V}\right)^{-1}$ is invertible, because $\left\|M_{V}\right\|<1$. Thus $\Gamma_{\Psi_{1,2}} u=0$. In other words, $\Psi_{1,2}(\lambda) u=0$ for each $\lambda \in \mathbb{D}$. This implies that $H_{\widetilde{V}}(\lambda) u=\Psi_{2,2}(\lambda) u$ for any $\widetilde{V} \in \mathbf{S}\left(\mathcal{E}, \mathcal{E}^{\prime}\right)$ and $\lambda \in \mathbb{D}$. Then $\Gamma_{H_{\tilde{V}}} u=\Gamma_{\Psi_{2,2}} u=\Gamma_{H_{V}} u$, and thus $\left\|\Gamma_{H_{\widetilde{V}}} u\right\|=$ $\left\|\Gamma_{H_{V}} u\right\|=\|u\|$. Hence $u \in \operatorname{Ker} D_{\Gamma_{H_{\tilde{V}}}}$ for any $\widetilde{V} \in \mathbf{S}\left(\mathcal{E}, \mathcal{E}^{\prime}\right)$.

Note that (1) is just the contrapositive of (2). To see that (2) holds, we use a similar argument as in the proof of (3). Assume we have a $V \in \mathbf{S}_{0}\left(\mathcal{E}, \mathcal{E}^{\prime}\right)$ with $\left\|\Gamma_{H_{V}}\right\|=1$. Let $u_{n}, n=0,1, \ldots$, be a sequence with $\left\|u_{n}\right\|=1$ and $\left\|\Gamma_{H_{V}} u_{n}\right\| \rightarrow 1$. Since $K_{V}$ in Proposition 4.3 is contractive, $-D_{M_{V}}\left(I-M_{\Psi_{1,1}} M_{V}\right)^{-1} \Gamma_{\Psi_{1,2}} u_{n} \rightarrow 0$, and thus $\Gamma_{\Psi_{1,2}} u_{n} \rightarrow 0$; again because $D_{M_{V}}\left(I-M_{\Psi_{1,1}} M_{V}\right)^{-1}$ is invertible. Now let $\widetilde{V}$ be an 
arbitrary function in $\mathbf{S}_{0}\left(\mathcal{E}, \mathcal{E}^{\prime}\right)$. Using that $\Gamma_{H_{\tilde{V}}}$ and $\Gamma_{H_{V}}$ can be written explicitly as in (4.3), it follows that $\lim _{n \rightarrow \infty} \Gamma_{H_{\widetilde{V}}} u_{n}=\lim _{n \rightarrow \infty} \Gamma_{\Psi_{2,2}} u_{n}=\lim _{n \rightarrow \infty} \Gamma_{H_{V}} u_{n}$. In particular, $\left\|\Gamma_{H_{\widetilde{V}}} u_{n}\right\| \rightarrow 1$. Thus $\left\|\Gamma_{H_{\widetilde{V}}}\right\|=1$.

Proof of Theorem 0.6 Assume there exists a $\widetilde{V} \in \mathbf{S}_{0}\left(\mathcal{G}, \mathcal{D}_{\omega^{*}}\right)$ with $\left\|B_{\widetilde{V}}\right\|<1$. Let $H_{\widetilde{V}}=\mathscr{R}_{\Phi}[\widetilde{V}]$, with $\Phi$ defined by (0.9). Then both $A$ and $\Gamma_{H_{\widetilde{V}}}$ are strict contractions. By Theorem 6.1 it then follows for any $V \in \mathbf{S}_{0}\left(\mathcal{G}, \mathcal{D}_{\omega^{*}}\right)$ that the operator $\Gamma_{H_{V}}$ is a strict contraction, and thus that $B_{V}$ is a strict contraction. This proves Part 1. Part 2 follows because it is the contrapositive of Part 1.

To see that the third statement holds, note that for any $V \in \mathbf{S}\left(\mathcal{G}, \mathcal{D}_{\omega^{*}}\right)$ the contractive interpolant $B_{V}$ in (0.11) satisfies

$$
\left\|D_{V} h\right\|=\left\|D_{\Gamma_{H_{V}}} D_{A} h\right\| \quad(h \in \mathcal{H}) .
$$

Thus $h \in \mathcal{H}$ is in $\operatorname{Ker} D_{V}$ if and only if $D_{A} h$ is in $\operatorname{Ker} D_{\Gamma_{V}}$. The statement then follows by applying Part 3 of Theorem 6.1 to the Redheffer representation $\mathscr{R}_{\Phi}$.

We conclude with the some bounds on $\left\|\Gamma_{H_{V}}\right\|$ for $V \in \mathbf{S}_{0}\left(\mathcal{E}, \mathcal{E}^{\prime}\right)$.

Proposition 6.2 Let $\Psi_{1,1}, \Psi_{1,2}, \Psi_{2,1}$ and $\Psi_{2,2}$ be as in (4.1) with $\Psi_{1,1}(0)=0$ and assume that the coefficient matrix (4.2) is contractive. For any $V \in \mathbf{S}_{0}\left(\mathcal{E}, \mathcal{E}^{\prime}\right)$ and $u \in \mathcal{U}$ we have

$$
\left\|\Gamma_{H_{V}} u\right\|^{2} \leq\|u\|^{2}-\frac{1-\left\|M_{V}\right\|^{2}}{\left\|I-M_{\Psi_{1,1}} M_{V}\right\|^{2}}\left\|\Gamma_{\Psi_{1,2}} u\right\|^{2} .
$$

In particular,

$$
\left\|\Gamma_{H_{V}} u\right\|^{2} \leq\|u\|^{2}-\frac{1-\left\|M_{V}\right\|}{1+\left\|M_{V}\right\|}\left\|\Gamma_{\Psi_{1,2}} u\right\|^{2} .
$$

If, in addition, the coefficient matrix (4.2) is an isometry, then

$$
\left\|\Gamma_{H_{V}} u\right\|^{2} \leq \frac{2\left\|M_{V}\right\|}{1+\left\|M_{V}\right\|}\|u\|^{2}+\frac{1-\left\|M_{V}\right\|}{1+\left\|M_{V}\right\|}\left\|\Gamma_{\Psi_{2,2}} u\right\|^{2} .
$$

Proof From the fact that the operator matrix $K_{V}$ in (4.5) is contractive we obtain

$$
\left\|\Gamma_{H_{V}} u\right\|^{2} \leq\|u\|^{2}-\left\|D_{V}\left(I-M_{\Psi_{11}} M_{V}\right)^{-1} \Gamma_{\Psi_{1,2}} u\right\|^{2} .
$$

Using that for an invertible operator $T \in \mathcal{L}(\mathcal{H})$ and $h \in \mathcal{H}$ we have the inequality $\|T h\| \geq\left\|T^{-1}\right\|^{-1}\|h\|$, it follows that

$$
\left\|\Gamma_{H_{V}} u\right\|^{2} \leq\|u\|^{2}-\left\|D_{V}^{-1}\right\|^{-2}\left\|\left(I-M_{\Psi_{11}} M_{V}\right)\right\|^{-2}\left\|\Gamma_{\Psi_{1,2}} u\right\|^{2} .
$$

The first inequality for $\left\|\Gamma_{H_{V}} u\right\|$ holds because $\left\|D_{M_{V}}^{-1}\right\| \leq\left(1-\left\|M_{V}\right\|^{2}\right)^{-\frac{1}{2}}$. Indeed, for any $h \in H^{2}(\mathcal{G})$ we have $\left\|D_{M_{V}} h\right\|^{2}=\|h\|^{2}-\left\|M_{V} h\right\|^{2} \geq\left(1-\left\|M_{V}\right\|^{2}\right)\|h\|^{2}$. So for $h=D_{M_{V}}^{-1} k$ we obtain $\|k\|^{2} \geq\left(1-\left\|M_{V}\right\|^{2}\right)\left\|D_{M_{V}}^{-1} k\right\|^{2}$, which proves our claim. 
To see that the second inequality holds, we use

$$
\left\|I-M_{\Psi_{1,1}} M_{V}\right\| \leq 1+\left\|M_{\Psi_{1,1}} M_{V}\right\| \leq 1+\left\|M_{V}\right\|,
$$

in order to obtain

$$
\frac{1-\left\|M_{V}\right\|^{2}}{\left\|I-M_{\Psi_{1,1}} M_{V}\right\|^{2}} \geq \frac{1-\left\|M_{V}\right\|^{2}}{\left(1+\left\|M_{V}\right\|\right)^{2}}=\frac{1-\left\|M_{V}\right\|}{1+\left\|M_{V}\right\|} .
$$

In case the coefficient matrix $K_{0}$ is an isometry, $\left\|\Gamma_{\Psi_{1,2}} u\right\|^{2}=\|u\|^{2}-\left\|\Gamma_{\Psi_{2,2}} u\right\|^{2}$. Hence, starting from the second inequality,

$$
\begin{aligned}
\left\|\Gamma_{H_{V}} u\right\|^{2} & \leq\|u\|^{2}-\frac{1-\left\|M_{V}\right\|}{1+\left\|M_{V}\right\|}\left(\|u\|^{2}-\left\|\Gamma_{\Psi_{2,2}} u\right\|^{2}\right) \\
& =\frac{2\left\|M_{V}\right\|}{1+\left\|M_{V}\right\|}\|u\|^{2}+\frac{1-\left\|M_{V}\right\|}{1+\left\|M_{V}\right\|}\left\|\Gamma_{\Psi_{2,2}} u\right\|^{2} .
\end{aligned}
$$

Open Access This article is distributed under the terms of the Creative Commons Attribution Noncommercial License which permits any noncommercial use, distribution, and reproduction in any medium, provided the original author(s) and source are credited.

\section{References}

1. Andô, T.: De Branges Spaces and Analytic Functions. Lecture Notes of the Division of Applied Mathematics Research Institute of Applied Electricity. Hokkaido University, Sapporo (1990)

2. Arov, D.Z., Dym, H.: J-contractive matrix-valued functions and related topics. Encyclopedia of Mathematics and its Applications, vol. 116. Cambridge University Press, Cambridge (2008)

3. Adamjan, V.M., Arov, D.Z., Kreĭn, M.G.: Infinite Hankel matrices and generalized Carathéodory-Fejér and I. Schur problems, (Russian), Funkcional. Anal. i Priložen. 2, (1968), no. 4, 1-17; English trans. Funct. Anal. Appl. 2, 269-281 (1968)

4. Adamjan, V.M., Arov, D.Z., Kreĭn, M.G.: Infinite Hankel block matrices and related extension problems (Russian), Izv. Akad. Nauk Armjan. SSR Ser. Mat. 6 (1971); English trans. Am. Math. Soc. Transl. 111, 133-156 (1978)

5. Arov, D.Z.: $\gamma$-generating matrices, $j$-inner matrix-functions and related extrapolation problems (Russian), Teor. Funktsiř Funktsional. Anal. i Priložen. 51 (1989), 61-67; English trans. J. Sov. Math. 52, 3487-3491 (1990)

6. Arov, D.Z.: Regular $J$-inner matrix-functions and related continuation problems. In: Linear Operators in Function Spaces (Timişoara, Romania, 1988). OT, vol. 43, pp. 63-87. Birkhäuser Verlag, Basel (1990)

7. Ball, J.A., Kheifets, A.: The inverse commutant lifting problem: characterization of associated Redheffer linear-fractional maps (submitted)

8. Biswas, A.: A harmonic-type maximal principle in commutant lifting. Integral Equ. Oper. Theory 28, 373-381 (1997)

9. Biswas, A., Foias, C., Frazho, A.E.: Weighted commutant lifting. Acta Sci. Math. (Szeged) 65, 657-686 (1999)

10. Douglas, R.G.: On majorization, factorization, and range inclusion of operators on Hilbert space. Proc. Am. Math. Soc. 17, 413-415 (1966)

11. Dzung, D., Bhosri, W., Frazho, A.E.: Mixed norm low order multirate filterbank design: relaxed commutant lifting approach. In: Proceedings of the 46th Annual Allerton Conference on Communication, Control, and Computing, pp. 452-459 (2008) 
12. Foias, C., Frazho, A.E.: The Commutant Lifting Approach to Interpolation Problems. OT, vol. 44. Birkhäuser-Verlag, Basel (1990)

13. Foias, C., Frazho, A.E., Gohberg, I., Kaashoek, M.A.: Metric Constrained Interpolation, Commutant Lifting and Systems. OT, vol. 100. Birkhäuser-Verlag, Basel (1998)

14. Foias, C., Frazho, A.E., Kaashoek, M.A.: Relaxation of metric constrained interpolation and a new lifting theorem. Integral Equ. Oper. Theory 42, 253-310 (2002)

15. Francis, B.A.: A Course in $H_{\infty}$ Control Theory. Lecture Notes in Control and Information Sciences, vol. 88. Springer, Berlin (1987)

16. Frazho, A.E., ter Horst, S., Kaashoek, M.A.: Coupling and relaxed commutant lifting. Integral Equ. Oper. Theory 54, 33-67 (2006)

17. Frazho, A.E., ter Horst, S., Kaashoek, M.A.: All solutions to the relaxed commutant lifting problem. Acta Sci. Math. (Szeged) 72, 299-318 (2006)

18. Frazho, A.E., ter Horst, S., Kaashoek, M.A.: Relaxed commutant lifting: An equivalent version and a new application, In: Recent Advances in Operator Theory (Seoul, South Korea, 2006) OT 187, pp. 157-168. Birkhäuser Verlag, Basel (2008)

19. Fuhrmann, P.A.: Linear Systems and Operators in Hilbert Space. McGraw-Hill, New York (1981)

20. ter Horst, S.: Relaxed commutant lifting and Nehari interpolation. Ph.D. Thesis, Vrije Universiteit, Amsterdam (2007). http://www.darenet.nl

21. ter Horst, S.: Relaxed commutant lifting: existence of a unique solution. Proc. Am. Math. Soc. 137, 2697-2707 (2009)

22. ter Horst, S.: Relaxed commutant lifting and a relaxed Nehari problem: Redheffer state space formulas. Math. Nachr. 282, 1753-1769 (2009)

23. Kheifets, A.: On a necessary but not sufficient condition for a $\gamma$-generating pair to be a Nehari pair. Integral Equations Operator Theory 21, 334-341 (1995)

24. Kheifets, A.: On regularization of $\gamma$-generating pairs. J. Funct. Anal. 130, 310-333 (1995)

25. Li, W.S., Timotin, D.: The relaxed intertwining lifting in the coupling approach. Integral Equ. Oper. Theory 54, 97-111 (2006)

26. Sz.-Nagy, B., Foias, C.: Dilation des commutants d'opérateurs. C. R. Acad. Sci. Paris Ser. A 266, 493-495 (1968)

27. Parrott, S.: On a quotient norm and the Sz-Nagy-Foias lifting theorem. J. Funct. Anal. 30, 311-328 (1978)

28. Redheffer, R.M.: On certain linear fractional representations. J. Math. Phys. 39, 269-286 (1960)

29. Redheffer, R.M.: On the relation of transmission-line theory to scattering and transfer. J. Math. Phys. 41, 1-41 (1962)

30. Sarason, D.: Generalized interpolation in $H^{\infty}$. Trans. Am. Math. Soc. 127, 179-203 (1967)

31. Treil, S., Volberg, A.: A fixed point approach to Nehari's problem and its applications, In: Toeplitz Operators and Related Topics (Santa Cruz, CA, 1992), The Harold Widom Anniversary Volume. OT, vol. 71, pp. 165-186, Birkhäuser Verlag, Basel (1994)

32. Volberg, A., Yuditskii, P.: Remarks on Nehari's problem, matrix $A_{2}$ condition, and weighted bounded mean oscillation. In: Linear and Complex Analysis. American Mathematical Society Translation Series 2, vol. 226, pp. 239-254. American Mathematical Society, Providence (2009)

33. Zhou, K., Doyle, J.C., Glover, K.: Robust and Optimal Control. Prentice-Hall, Upper Saddle River (1996) 